\title{
Effects of rotation on the evolution of primordial stars
}

\author{
S. Ekström ${ }^{1}$, G. Meynet $^{1}$, C. Chiappini ${ }^{1,2}$, R. Hirschi ${ }^{3}$, and A. Maeder ${ }^{1}$ \\ 1 Geneva Observatory, University of Geneva, Maillettes 51, 1290 Sauverny, Switzerland \\ e-mail: Sylvia.Ekstrom@obs.unige.ch \\ 2 Osservatorio Astronomico di Trieste, via G.B. Tiepolo 11, 34131 Trieste, Italy \\ 3 University of Keele, Keele ST5 5BG, UK
}

Received 22 February 2008 / Accepted 1 July 2008

\begin{abstract}
Context. Although still beyond our observational abilities, Population III stars are interesting objects from many perspectives. They are responsible for the re-ionisation of the inter-galactic medium. They also left their chemical imprint in the early Universe, which can be deciphered in the most metal-poor stars in the halo of our Galaxy.

Aims. Rotation has been shown to play a determinant role at very low metallicity, bringing heavy mass loss where almost none was expected. Is this still true when the metallicity strictly equals zero? The aim of our study is to answer this question, and to determine how rotation changes the evolution and the chemical signature of the primordial stars.

Methods. We have calculated seven differentially-rotating stellar models at zero metallicity, with masses between 9 and $200 M_{\odot}$. For each mass, we also calculated a corresponding model without rotation. The evolution is followed up to the pre-supernova stage.

Results. We find that $Z=0$ models rotate with an internal profile $\Omega(r)$ close to local angular momentum conservation, because of a very weak core-envelope coupling. Rotational mixing drives an $\mathrm{H}$-shell boost due to a sudden onset of the $\mathrm{CNO}$ cycle in the shell. This boost leads to a high ${ }^{14} \mathrm{~N}$ production, which can be as much as $10^{6}$ times higher than the production of the non-rotating models. Generally, the rotating models produce much more metal than their non-rotating counterparts. The mass loss is very low, even for the models that reach critical velocity during the main sequence. It may however have an impact on the chemical enrichment of the Universe, because some of the stars are supposed to collapse directly into black holes. They would contribute to the enrichment only through their winds. While in that case non-rotating stars would not contribute at all, rotating stars may leave an imprint on their surrounding. Due to the low mass loss and the weak coupling, the core retains a high angular momentum at the end of the evolution. The high rotation rate at death probably leads to a much stronger explosion than previously expected, changing the fate of the models. The inclusion of our yields in a chemical evolution model of the Galactic halo predicts log values of N/O, $\mathrm{C} / \mathrm{O}$ and ${ }^{12} \mathrm{C} /{ }^{13} \mathrm{C}$ ratios of $-2.2,-0.95$ and 50 respectively at $\log \mathrm{O} / \mathrm{H}+12=4.2$.
\end{abstract}

Key words. stars: evolution - stars: rotation - stars: chemically peculiar - stars: supernovae: general

\section{Introduction}

Population III (PopIII) stars occupy a key position in the evolution of the Universe. They are the first sources of light that re-ionised the Universe. Also, they are the first producers of the heavy elements in the early Universe ${ }^{1}$.

Because of the peculiar conditions of the early Universe, especially the lack of coolants provided by the metals, the very first metal-free stars that formed are supposed to be very massive, with a typical mass $M \approx 10^{2}-10^{3} M_{\odot}$ (Abel et al. 2002; Bromm et al. 2002). Nakamura \& Umemura (2001) propose a bimodal IMF, with one peak at $M \approx 10^{2} M_{\odot}$ and the other at $M \approx 1 M_{\odot}$. The bimodality is due to a threshold in the initial density of the cloud. Since this first suggestion that PopIII may not all be very massive objects, several studies have shown that the radiation field of a pre-existing star has a strong impact on subsequent star formation (Omukai \& Yoshii 2003; O'Shea et al. 2005; Greif \& Bromm 2006). This would allow lowermass metal-free stars to appear. Moreover Silk \& Langer (2006) showed that even if the early Universe was free of magnetic fields, magnetic seeds can be generated in the disc surrounding

\footnotetext{
1 According to the standard Big Bang Nucleosynthesis model, only elements with nucleon number $A \leq 7$ were formed at a significant level during the first minutes of the Universe (e.g. Iocco et al. 2007).
}

an accreting stellar progenitor. These seeds can be amplified through a dynamo process and become strong enough to play an important role in the accretion rate. This again would reduce substantially the final mass range of the first stars. Though it was once suggested that the most metal-poor stars known to this day may be low-mass PopIII that have undergone surface pollution (Shigeyama et al. 2003), it is now widely accepted that metal-free stars were too massive to still be present nowadays $\left(M>0.8 M_{\odot}\right)$. The observation facilities are not yet able to reach far enough for a direct detection (except maybe for the detection of pair-instability supernovae at intermediate redshifts, see Scannapieco et al. 2005). The only way to constrain their physical properties is indirect observation of their chemical or radiative signature. Although it is not firmly established whether the most metal-deficient stars of the halo of our Galaxy are truly second generation stars (Salvadori et al. 2007), most authors consider that they are born from a cloud enriched by only a few primordial progenitors (Christlieb et al. 2002; Umeda \& Nomoto 2003). Recently, accurate measurements of their surface abundances have started to allow some constraints to be placed on the theoretical results of PopIII evolution (Cayrel et al. 2004; Barklem et al. 2005; Cohen et al. 2007; Aoki et al. 2007).

In view of their importance, PopIII stars have been the subject of many evolutionary calculations. Amongst the latest ones, most present the evolution until the AGB phase or central C 
ignition (Chieffi et al. 2001; Marigo et al. 2001, 2003; Siess et al. 2002; Gil-Pons et al. 2007; Lau et al. 2008). Others are focused on the final stages (Heger \& Woosley 2002; Umeda \& Nomoto 2005). To our knowledge, there are only two studies that include rotating models: the one by Marigo et al. (2003), and the one by Heger et al. (2000b). Marigo et al. (2003) study very massive objects with masses between 120 and $1000 M_{\odot}$. In their work, rotating stars are treated as rigid bodies and the effects of rotation intervene mainly through a correction factor applied on the radiative mass loss (see Sect. 2.2). In these models, no rotational mixing is accounted for. Heger et al. (2000b) follow the evolution of differentially-rotating stellar models of $15-250 M_{\odot}$ from the zero-age main sequence (ZAMS) to core collapse, but the detailed results of these models have not been published.

The present paper explores the effects of differential rotation on primordial massive stars. We think it is worth doing such a study for two main reasons. First, for metallicities between 0.004 and 0.040 , the inclusion of the effects of differential rotation in stellar evolution codes has allowed us to improve the agreement between the models and observations in many aspects: it has allowed us to better reproduce the observed surface abundances (Heger \& Langer 2000; Meynet \& Maeder 2000), the ratio of blue-to-red supergiants at low metallicity (Maeder \& Meynet 2001 ), the variation with metallicity of the WR/O ratios and of the numbers of type Ibc to type II supernovae (Meynet \& Maeder $2003,2005)$. It is interesting to see what a code that has been successfully compared with observations can predict in a metallicity domain where no direct observations are available. Second, rotation has been shown to have important effects on low metallicity stars (Meynet et al. 2006). During the Main Sequence (MS), the radiative winds are very low because of the lack of metals, so the star cannot lose its angular momentum. If it starts its evolution with a sufficiently high equatorial velocity, it may reach the critical limit. In that case, the outer layers are no longer bound and some mass is lost, probably through a decretion disc (Owocki 2005) that may be quickly erased by the radiation. Further in the evolution, the rotational mixing brings freshly synthesised metals to the surface. The opacity of the outer layers are enhanced and the radiative mass loss is increased. While low metallicity stars were supposed to lose almost no mass at all, it has been shown that fast rotation can dramatically enhance the mass loss and thus modify drastically the evolution of these stars. How does a total absence of metals alter the above picture? It is interesting to extend the study to strictly $Z=0$ models.

The paper is organized as follow: in Sect. 2, we describe the physical ingredients of the models; Sect. 3 is dedicated to the presentation of the evolution of the models; in Sect. 4, we present the pre-SN structure and the yields obtained; we study the effect of their inclusion in a galactic chemical evolution code in Sect. 5, and then a discussion follows in Sect. 6.

Electronic tables of the evolutionary sequences and the final structure files are available at http://obswww . unige.ch/ Recherche/evol/Rotating-primordial-stars

\section{Physical ingredients of the models}

The stellar models presented here have masses ranging from 9 to $200 M_{\odot}$. The initial composition is $X=0.76, Y=0.24$ and $Z=0$. The models with masses between 25 and $85 M_{\odot}$ were computed until the end of the hydrostatic core Si burning. The $9 M_{\odot}$ model developed a strongly degenerated core before carbon ignition and was thus stopped then. The $15 M_{\odot}$ model was stopped at the end of oxygen burning also because of a too
Table 1. Rotational properties of the models. The masses are in solar mass units, and the velocities in $\mathrm{km} \mathrm{s}^{-1}$. The last column gives the central hydrogen mass fraction when the model reaches the critical limit.

\begin{tabular}{rrrrr}
\hline \hline Mass & $v_{\text {ini }}$ & $v / v_{\text {crit }}$ & $\bar{v}(\mathrm{MS})$ & $X_{\mathrm{c}, \mathrm{b}}$ \\
\hline 9 & 500 & 0.54 & 430 & - \\
15 & 800 & 0.72 & 626 & 0.018 \\
25 & 800 & 0.63 & 657 & 0.253 \\
40 & 800 & 0.56 & 700 & 0.326 \\
60 & 800 & 0.52 & 740 & 0.360 \\
85 & 800 & 0.48 & 754 & 0.399 \\
200 & 800 & 0.40 & 733 & 0.428 \\
\hline
\end{tabular}

degenerate core at that time. Because of numerical difficulties, the $200 M_{\odot}$ was stopped shortly after central He exhaustion.

The computation was carried out with the Geneva stellar evolution code. The version of the code is the one used in Hirschi et al. (2004) that includes the necessary implementations to continue through advanced stages up to the pre-SN. It has been slightly modified to join the reaction networks of $\mathrm{H}$ and $\mathrm{He}$ burning during the MS, as required for zero-metallicity models.

The reaction rates are taken from the NACRE database (http://pntpm.ulb.ac.be/Nacre/barre_database.

htm). The opacities come from Iglesias \& Rogers (1996), complemented at low temperature by the molecular opacities of Alexander \& Ferguson (1994). The treatment of convective instability is done according to the Schwarzschild criterion. As in Hirschi (2007), convection is treated as a diffusive process from oxygen burning onwards. An overshooting parameter $\alpha_{\text {over }}=0.2 H_{\mathrm{P}}$ is used for $\mathrm{H}$ - and He-burning cores only.

\subsection{Rotation}

The effects of rotation are computed in the framework of the Roche model (see Ekström et al. 2008b) and according to the shellular-rotation hypothesis (see Zahn 1992). The code couples the centrifugal force to the internal structure equations (Maeder \& Meynet 2000b). This modifies the hydrostatic equilibrium and the surface conditions. The rotation-induced instabilities taken into account are the meridional circulation, the secular and dynamical shears.

- The meridional circulation arises from the impossibility for a rotating star to be in hydrostatic and radiative equilibrium at the same time (the von Zeipel theorem). The vertical component of the meridional circulation velocity is $u(r, \theta)=$ $U(r) P_{2}(\cos \theta)$ with $P_{2}(x)$ the second Legendre polynomial. The formulation of the radial amplitude $U(r)$ has been determined by Zahn (1992) and Maeder \& Zahn (1998) as:

$$
\begin{aligned}
U(r)= & \frac{P}{\rho g C_{P} T\left[\nabla_{\mathrm{ad}}-\nabla_{\mathrm{rad}}+(\varphi / \delta) \nabla_{\mu}\right]} \\
& \cdot\left(\frac{L}{M_{\star}}\left[E_{\Omega}+E_{\mu}\right]+\frac{C_{P}}{\delta} \frac{\partial \Theta}{\partial t}\right)
\end{aligned}
$$

where $C_{P}$ is the specific heat, $\nabla_{\mathrm{ad}}=\frac{P \delta}{\rho T C_{P}}$ the adiabatic gradient, $M_{\star}=M\left(1-\frac{\Omega^{2}}{2 \pi g \rho_{\mathrm{m}}}\right)$, and $\Theta=\tilde{\rho} / \bar{\rho}$ is the ratio of the variation of the density to the average density on an equipotential. $\varphi$ and $\delta$ arise from the generic equation of state: $\frac{\mathrm{d} \rho}{\rho}=\alpha \frac{\mathrm{d} P}{P}+\varphi \frac{\mathrm{d} \mu}{\mu}-\delta \frac{\mathrm{d} T}{T} . E_{\Omega}$ and $E_{\mu}$ are terms that depend on the $\Omega$ - and $\mu$-distributions respectively (see the details of 
these expressions in Maeder \& Zahn 1998). $E_{\Omega}$ is the dominant term in the round brackets, and it can be reasonably approximated by: $E_{\Omega} \simeq \frac{8}{3}\left[1-\frac{\Omega^{2}}{2 \pi g \bar{\rho}}\right]\left(\frac{\Omega^{2} r^{3}}{G M}\right)$. The term in square brackets is known as the Gratton-Öpik term. It may become largely negative in the outermost layers of the stars, where $\bar{\rho}$ becomes very small, which leads to $U(r)<0$. In this case, the circulation descend along the polar axis from the surface inwards, and then rises in the equatorial plane outwards to the surface, bringing angular momentum to the surface.

- Differential rotation induces shear turbulence at the interface of layers having different rotational velocities. A layer remains stable if the excess kinetic energy due to the differential rotation does not overcome the buoyancy force. This is known as the Richardson criterion and is expressed as $R i=\frac{N^{2}}{(\partial u / \partial z)^{2}}>R i_{\mathrm{c}}$, where $u$ is the horizontal velocity, $z$ the vertical coordinate, and $N^{2}=\frac{g \delta}{H_{P}}\left(\nabla_{\mathrm{ad}}-\nabla+(\varphi / \delta) \nabla_{\mu}\right)$ is the Brunt-Väisälä frequency. $R i_{\mathrm{c}}=1 / 4$ is the critical value for stability. The coefficient of diffusion by dynamical shear implemented in the code is described in Hirschi et al. (2004):

$D_{\text {dyn }}=\frac{1}{3} r \Delta \Omega \Delta r$

where $r$ is the mean radius of the zone constituted of consecutive shells with $R i<R i_{\mathrm{c}}=1 / 4, \Delta \Omega$ is the variation of $\Omega$ over this zone and $\Delta r$ is the extend of the zone. The timescale for the dynamical shear is $\tau_{\text {dyn }}=\sqrt{r^{3} /\left(G m_{r}\right)}$. The prescription for $D_{\text {dyn }}$ is valid when the thermal adjustment timescale $\tau_{\text {th }}=\frac{d^{2}}{12 K}$ (for a sphere with diameter $d$ and a thermal diffusivity $K$ ) is longer than the dynamical timescale.

- Secular shear arises when the thermal dissipation reduces the buoyancy force. The coefficient of diffusion by secular shear turbulence was determined by Maeder (1997) as:

$$
\begin{aligned}
D_{\text {shear }}= & \frac{4\left(K+D_{\mathrm{h}}\right)}{\left[\frac{\varphi}{\delta} \nabla_{\mu}\left(1+\frac{K}{D_{\mathrm{h}}}\right)+\left(\nabla_{\mathrm{ad}}-\nabla_{\mathrm{rad}}\right)\right]} \\
& \cdot \frac{H_{P}}{g \delta}\left[\frac{\alpha}{4}\left(0.8836 \Omega \frac{\mathrm{d} \ln \Omega}{\mathrm{d} \ln r}\right)^{2}-\left(\nabla^{\prime}-\nabla\right)\right]
\end{aligned}
$$

with $K=\frac{4 a c T^{3}}{3 \kappa \rho^{2} C_{P}}$ the thermal diffusivity, $\alpha$ the fraction of the excess energy in the shear that will contribute to mixing, and $\left(\nabla^{\prime}-\nabla\right)$ the difference between the internal nonadiabatic gradient and the local gradient. The latter term is extremely small and is neglected in the calculation. $D_{\mathrm{h}}$ is the coefficient for the horizontal turbulence. It is determined as in Zahn (1992): $D_{\mathrm{h}}=\frac{1}{c_{\mathrm{h}}} r|2 V(r)-\alpha U(r)|$, where $c_{\mathrm{h}}$ is a constant of order $1, V(r)$ is the horizontal component of the meridional circulation velocity, $U(r)$ its vertical component, and in this expression $\alpha=\frac{1}{2} \frac{\mathrm{d} \ln \left(r^{2} \bar{\Omega}\right)}{\mathrm{d} \ln r}$.

In the zones where $R i<R i_{\mathrm{c}}$, the shear mixing coefficient is eventually set to:

$D_{\text {shear }}=D_{\text {dyn }}+D_{\text {shear }}$.

The transport of angular momentum inside a star is implemented following the prescription of Zahn (1992), complemented by the works of Talon \& Zahn (1997) and Maeder \& Zahn (1998). In the radial direction, it obeys the following equation:

$\rho \frac{\mathrm{d}}{\mathrm{d} t}\left(r^{2} \bar{\Omega}\right)_{M_{r}}=\frac{1}{5 r^{2}} \frac{\partial}{\partial r}\left(\rho r^{4} \bar{\Omega} U(r)\right)+\frac{1}{r^{2}} \frac{\partial}{\partial r}\left(\rho D r^{4} \frac{\partial \bar{\Omega}}{\partial r}\right)$.
The first term in the right hand side of this equation is the divergence of the advected flux of angular momentum, while the second term is the divergence of the diffused flux. The coefficient $D$ is the total diffusion coefficient, taking into account the various instabilities which transport angular momentum (convection, shear). After the end of the MS, the timescale of the evolution shortens, so only the diffusive term is kept in the formulation of the transport of the angular momentum.

The transport of chemical elements by the meridional circulation has been shown to be satisfactorily approximated by a diffusion process (see Chaboyer \& Zahn 1992) throughout the whole evolution.

Since very low- or zero-metallicity stars are more compact than their metal-rich counterparts, a similar ratio of the initial velocity to the critical velocity ${ }^{2} v / v_{\text {crit }}$ leads to a higher equatorial velocity. In order to explore the effects of rotation on metalfree models, we chose a high initial velocity, $800 \mathrm{~km} \mathrm{~s}^{-1}$. Note that for the $60 M_{\odot}$ model, this initial velocity corresponds to a total angular momentum content that is similar to a solar metallicity model of the same mass rotating at $300 \mathrm{~km} \mathrm{~s}^{-1}$. The $9 M_{\odot}$ model was computed with a lower initial velocity $\left(500 \mathrm{~km} \mathrm{~s}^{-1}\right)$ because $800 \mathrm{~km} \mathrm{~s}^{-1}$ is overcritical on the ZAMS for this model. Table 1 presents the initial equatorial velocities of the models, the corresponding $v / v_{\text {crit }}$ ratio and the mean velocity during MS.

In order to be able to compare our results with other works, we computed, for each mass, a corresponding model without rotation.

\subsection{Mass loss}

Zero-metallicity stars are supposed to evolve at constant mass due to a scaling of the wind with metallicity of the form $\dot{M} \propto\left(Z / Z_{\odot}\right)^{0.5}$. However, according to Kudritzki (2002), H and He lines are able to remove some mass if the luminosity is sufficiently high. We thus adopted the Kudritzki (2002) prescription for the models presenting a luminosity $\log \left(L / L_{\odot}\right)>6$. The 85 and $200 M_{\odot}$ models were computed with it from the start, while we turned it on during the MS evolution of the $60 M_{\odot}$. Since Kudritzki's prescription does not correspond to the strictly $Z=0$ case, we used the same adaptations as in Marigo et al. (2003): 1) the surface metallicity is set $Z=\max \left(Z_{\mathrm{min}}, Z_{\mathrm{eff}}\right)$, where $Z_{\min }=10^{-4} Z_{\odot}$ is the lowest value in the validity range of Kudritzki's prescription, and $Z_{\mathrm{eff}}=1-X-Y$ is the effective surface metallicity of the model (allowing for self-enrichment); and 2) the effective temperature is set $T_{\text {eff }}=\min \left(T_{\text {eff }}, 60000 \mathrm{~K}\right)$, i.e. we did not extrapolate Kudritzki's formula beyond its validity domain but constrained $T_{\text {eff }} \leq 60000 \mathrm{~K}$ for the calculation of the mass loss. The models with a mass lower than $60 M_{\odot}$ never reach $\log \left(L / L_{\odot}\right)>6$, so they were computed without radiative mass loss.

Rotation, by lowering the effective gravity at the surface, helps the radiation remove mass from the star. As shown in Maeder \& Meynet (2000a), the line-driven mass loss is enhanced by a factor which depends on the angular velocity such that the mass loss in the case of rotation becomes:

$$
\dot{M}(\Omega)=F_{\Omega} \cdot \dot{M}(\Omega=0) \text { where } F_{\Omega}=\frac{(1-\Gamma)^{\frac{1}{\alpha}-1}}{\left[1-\frac{\Omega^{2}}{2 \pi G \rho_{\mathrm{m}}}-\Gamma\right]^{\frac{1}{\alpha}-1}} .
$$

2 The critical velocity $v_{\text {crit }}$ is reached when the gravitational acceleration is exactly counterbalanced by the centrifugal force. In the framework of the Roche model, one has: $v_{\text {crit }}=\sqrt{\frac{2}{3} \frac{G M}{R_{\mathrm{pb}}}}$, where $R_{\mathrm{pb}}$ is the polar radius at break-up limit. 

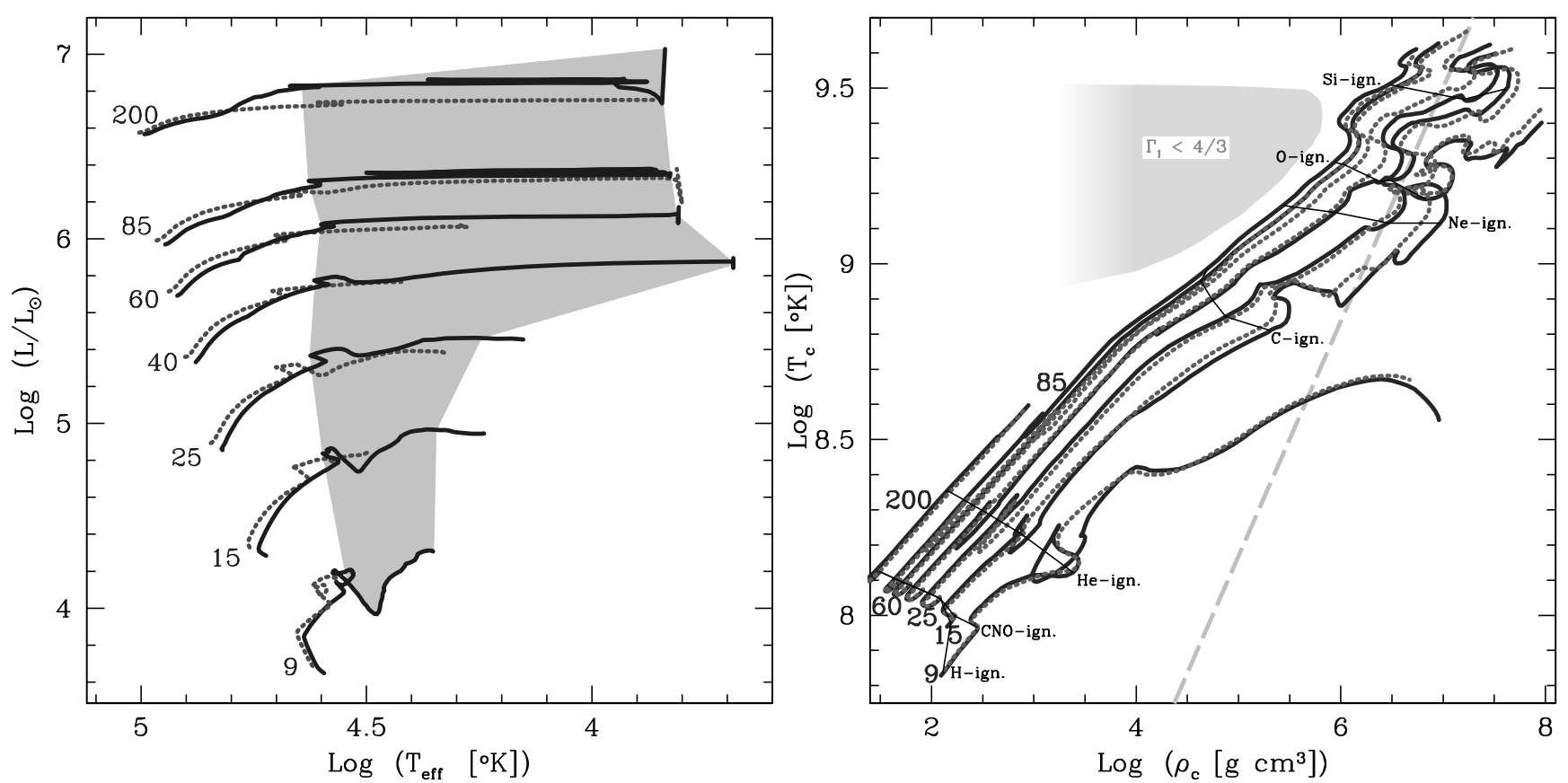

Fig. 1. Evolution of $Z=0$ models (with rotation: solid lines; without rotation: dotted lines). Left: Hertzsprung Russell diagram. The grey area shows the zone of the diagram where He burns in the core of the rotating models. Right: central temperature vs. central density diagram. The ignition of the different burning stages are given for the rotating models. The grey area indicates the zone of the diagram where the pair-creation instability occurs. The dashed grey line shows the locus of the points where the perfect gas pressure is equal to the completely-degenerate non-relativistic pressure.

We see that this expression depends also on the Eddington factor $\Gamma=L / L_{\mathrm{Edd}}=\kappa L /(4 \pi c G M)$ where $\kappa$ is the electron-scattering opacity.

Moreover, there is another situation in which the models can lose mass: it is when the external layers of the star reach the critical velocity. The over-critical layers are no longer bound to the star, although it is not yet clear whether they are expelled in the interstellar medium or remain in the surroundings, perhaps in the form of a disc. However, the removal of these layers brings the surface back to sub-critical velocity until the evolution of the star accelerates it again. In the present calculation, as in Meynet et al. (2006), once the model reaches the break-up limit, we adapted the mass loss rate to keep the surface just under the critical velocity. This treatment was applied to all the models except the $9 M_{\odot}$, which never reached the critical limit.

\section{Evolution}

\subsection{Hertzsprung Russell diagram and central conditions}

In the left panel of Fig. 1, we present the Hertzsprung Russell diagram (HRD) of our rotating and non-rotating models, and in the right panel, their tracks in the $\log T_{\mathrm{c}}-\log \rho_{\mathrm{c}}$ diagram. We notice the expected characteristics of rotating models: the ZAMS is shifted towards lower effective temperature and luminosity with respect to the non-rotating case ${ }^{3}$. Then, when the evolution proceeds, the tracks become more luminous, and the main-sequence turn-off is shifted to cooler temperature: the core of the rotating models is refuelled by fresh $\mathrm{H}$ brought by the mixing. It thus grows, leading to an enhancement of the luminosity.

The 9 and $15 M_{\odot}$ models are powered only by $p p$-chains when they arrive on the ZAMS and thus they continue their

\footnotetext{
3 This shift is due to the sustaining effect of the rotation: the gravity is counter-balanced both by the gas pressure and the centrifugal force in such a way that the star behaves like a lower mass one.
}

initial contraction. It is only when they have produced a sufficient amount of carbon through the $3 \alpha$ reaction (about $10^{-12}$ in mass fraction) that the $\mathrm{CNO}$ cycle can start, drawing a hook in the $\log T_{\mathrm{c}}-\log \rho_{\mathrm{c}}$ diagram. The onset of the CNO cycle in these models can also be seen in the HRD: their tracks evolve towards the blue side of the diagram, until the energy provided by the CNO cycle stops the contraction and bends the tracks back into the usual MS feature. In the rotating $9 M_{\odot}$ model, this happens at an age of $12.2 \mathrm{Myr}$ (when the central $\mathrm{H}$ mass fraction is $X_{\mathrm{c}}=0.439$ ) while in the non-rotating one it happens a little earlier, at an age of $10.9 \mathrm{Myr}$ (but at a similar burning stage: $\left.X_{\mathrm{c}}=0.439\right)$. In the case of the non-rotating $15 M_{\odot}$ model, it happens after merely $1.5 \mathrm{Myr}\left(X_{\mathrm{c}}=0.695\right)$, while it takes $2 \mathrm{Myr}$ $\left(X_{\mathrm{c}}=0.677\right)$ in the case of the rotating one. Marigo et al. (2001) find that the mass limit for CNO ignition to occur on the ZAMS is $20 M_{\odot}$. Our results are consistent with that limit.

After central $\mathrm{H}$ exhaustion, the core He-burning phase (CHeB) starts right away: the core was already hot enough to burn a little He during the MS so it does not need to contract much further. The transition between core $\mathrm{H}$ burning and $\mathrm{He}$ burning is smooth because the model is continuously sustained by central nuclear burning. This prevents the models from starting a redward evolution, so they remain in the blue part of the $\mathrm{HRD}$ at the beginning of $\mathrm{CHeB}$. The H-burning shell is powered only by pp-chains and remains radiative. The main part of the luminosity is provided by the core (see in Fig. 2 left, the case of the rotating $15 M_{\odot}$ model). Something particular happens to the rotating models during the $\mathrm{CHeB}$ phase: because of rotational mixing, some carbon produced in the core is diffused towards the H-burning shell, allowing a sudden ignition of the $\mathrm{CNO}$ cycle in the shell. This boost of the shell leads to a retraction of the convective core and a decrease of the luminosity. At the same time, it transforms the quiet radiative H-burning shell into an active convective one (see Fig. 2 centre). Later, the model takes the structure of a higher metallicity one: a convective He-burning core, 

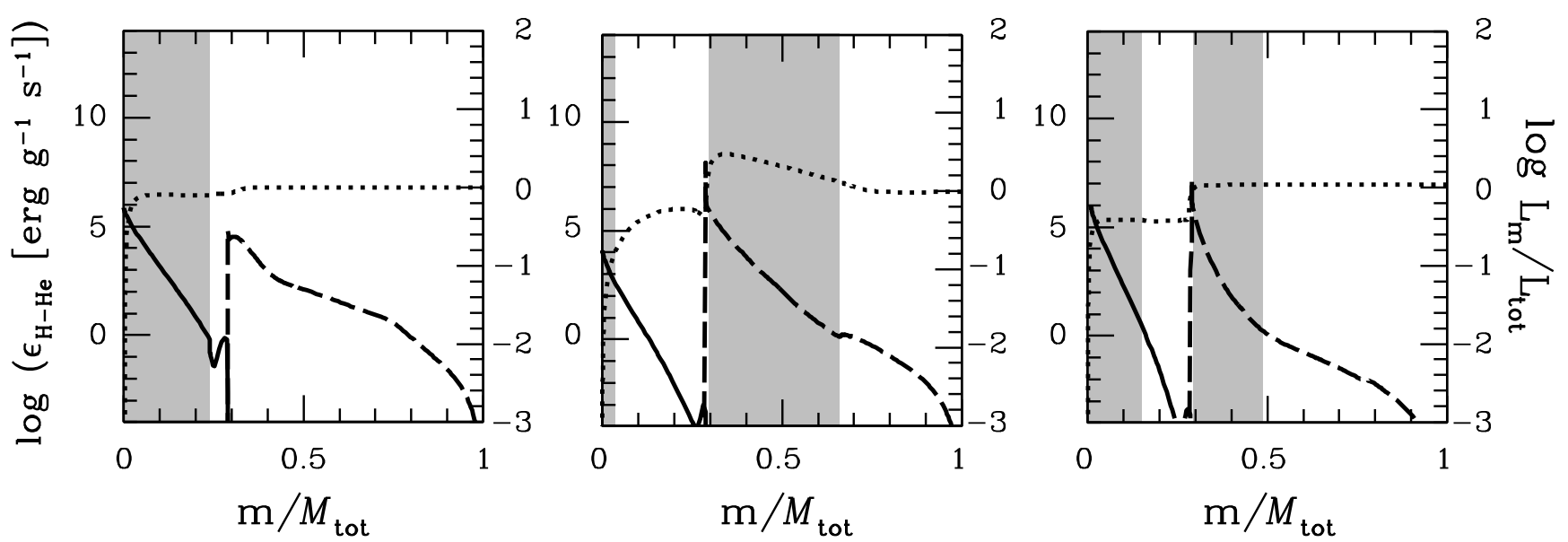

Fig. 2. Internal structure of the $15 M_{\odot}$ rotating model at different stages of the core He burning. The solid line is the He-burning energy production and the dashed line the H-burning energy production. The dotted line is the fraction of the total luminosity (scale on the right). The grey areas show the convective zones. Left: before the CNO shell boost (age: $13.739 \mathrm{Myr} ; Y_{\mathrm{c}}=0.4632$ ) - centre: at the moment of the boost (age: $13.744 \mathrm{Myr}$; $\left.Y_{\mathrm{c}}=0.4919\right)-$ right: after the boost (age: $\left.13.757 \mathrm{Myr} ; Y_{\mathrm{c}}=0.4840\right)$.

convective H-burning shell and expanding envelope (see Fig. 2 right). This boost appears in Fig. 1 (left) as a deep $V$-shaped feature in the rotating tracks of the 9 and $15 M_{\odot}$ models, and as a slight depression in the 25 and $40 M_{\odot}$ tracks: the luminosity decreases at the same time as the $T_{\text {eff }}$ cools down. The only non-rotating models that present the same feature are the 25 and $85 M_{\odot}$. In this case, the structure is such that the growing convective core reaches the bottom of the quiet radiative H-burning shell, triggering the same event as in the rotating models. Such a CNO boost leaves an imprint in the $\log T_{\mathrm{c}}-\log \rho_{\mathrm{c}}$ diagram: a second hook can be seen in the tracks, which takes the shape of a complex loop in the case of the $9 M_{\odot}$ track.

The crossing of the HRD is slow, unlike higher metallicity models. As we mentioned above, the nuclear burning is continuous inside the core, therefore the $Z=0$ models do not go through a phase of rapid core contraction and its mirror effect of fast envelope expansion. On the HRD, we note that the crossing of the diagram takes place during the whole $\mathrm{CHeB}$ (grey area).

In the non-rotating set, all the models with mass between 9 and $60 M_{\odot}$ end their life as blue supergiants (BSG), while the 85 and $200 M_{\odot}$ become red supergiants (RSG). This compares well with the tracks obtained by Marigo et al. (2001). As in their study, all the models with masses between 9 and 60 start and finish core He burning in the blue part of the HRD. Only the highest masses models $\left(M \geq 70 M_{\odot}\right)$ end their central He combustion on their Hayashi line.

Generally, the rotating tracks end their evolution in a cooler part of the HRD than their non-rotating counterparts. The only exception is the $85 M_{\odot}$, which ends its life as a BSG because of a blue loop after $\mathrm{CHeB}$. The rotating 15 and $25 M_{\odot}$ end their evolution in the blue part of the HRD, without any redward incursion. The rest of the rotating models become RSG. None of our models meet the conditions to be Wolf-Rayet stars. The $85 M_{\odot}$ star ends its life with a $\log T_{\text {eff }}$ higher than 4.3 , but its surface abundance of hydrogen remains too high.

\subsection{Lifetimes}

Table 2 shows the lifetimes for the MS and $\mathrm{CHeB}$ phases. During the MS, rotation induces an increase in the lifetime from $12.8 \%$ for the $200 M_{\odot}$ to $24.5 \%$ for the $15 M_{\odot}$ model. The models with higher initial $v / v_{\text {crit }}$ show a greater increase. For the models that
Table 2. Final masses, total lifetime, MS and core He-burning durations, masses of the He- and CO-core for our models at the pre-SN stage. Masses are in $M_{\odot}$, velocities in $\mathrm{km} \mathrm{s}^{-1}$ and times in Myr.

\begin{tabular}{rrrrrrrr}
\hline \hline Mass & $v_{\text {ini }}$ & $M_{\text {fin }}$ & $\tau_{\text {life }}$ & $\tau_{\mathrm{H}}$ & $\tau_{\mathrm{He}}$ & $M_{\mathrm{He}}$ & $M_{\mathrm{CO}}$ \\
\hline 9 & 0 & 9.00 & 22.0 & 19.50 & 1.99 & 1.73 & 0.46 \\
9 & 500 & 9.00 & 27.1 & 23.90 & 2.58 & 2.09 & 0.63 \\
15 & 0 & 15.00 & 11.6 & 10.60 & 0.83 & 3.62 & 2.92 \\
15 & 800 & 14.96 & 14.4 & 13.20 & 0.98 & 4.31 & 2.71 \\
25 & 0 & 25.00 & 7.29 & 6.64 & 0.56 & 7.51 & 5.39 \\
25 & 800 & 24.75 & 8.47 & 7.88 & 0.50 & 9.14 & 6.30 \\
40 & 0 & 40.00 & 5.03 & 4.60 & 0.36 & 15.95 & 14.52 \\
40 & 800 & 37.99 & 5.88 & 5.41 & 0.41 & 17.22 & 13.53 \\
60 & 0 & 59.99 & 3.96 & 3.60 & 0.30 & 26.52 & 25.11 \\
60 & 800 & 57.59 & 4.57 & 4.20 & 0.31 & 30.86 & 30.58 \\
85 & 0 & 84.93 & 3.42 & 3.05 & 0.31 & 34.77 & 34.50 \\
85 & 800 & 74.57 & 3.84 & 3.52 & 0.28 & 44.38 & 43.92 \\
200 & 0 & 199.95 & 2.53 & 2.27 & 0.23 & 101.63 & 95.69 \\
200 & 800 & 183.56 & 2.83 & 2.56 & 0.24 & 109.65 & 95.97 \\
\hline
\end{tabular}

reach the critical limit, we find an almost linear relation between the relative enhancement of the MS lifetime $\Delta \tau_{\mathrm{H}}$ and the initial ratio $v / v_{\text {crit }}$ of the form

$\Delta \tau_{\mathrm{H}}=\frac{\tau_{\mathrm{H}}\left(v / v_{\text {crit }}\right)-\tau_{\mathrm{H}}(0)}{\tau_{\mathrm{H}}(0)}=0.362 v / v_{\text {crit }}$

(when $\tau_{\mathrm{H}}$ is expressed in Myr) which reproduces the values of $\Delta \tau_{\mathrm{H}}$ obtained in our models with an accuracy better than $7 \%$.

For our non-rotating $9 M_{\odot}$, the MS lifetime is shorter by $12 \%$ than that found by Marigo et al. (2001), which is consistent with our lower overshoot parameter: the convective core of our model is about $10 \%$ smaller than theirs. They also have a higher initial hydrogen abundance $(X=0.77)$. Gil-Pons et al. (2007) provide two models of $9 M_{\odot}$, one computed without overshoot and the other with the overshoot theory presented by Eldridge \& Tout (2004). Their calculation of the convective boundaries could be approximated by $\alpha_{\mathrm{over}}=0.3 H_{\mathrm{P}}$ (Gil-Pons, priv. comm.). The lifetime of our model is about $21 \%$ shorter than the model they calculated with this higher overshoot. But it is also $11 \%$ shorter than their model calculated without any overshoot. In a previous paper (Gil-Pons et al. 2005), they show the MS evolution of the convective core and of the $\mathrm{H}$ and $\mathrm{He}$ abundances for this model. The transition from $p p$-chain burning to $\mathrm{CNO}$ cycle occurs 

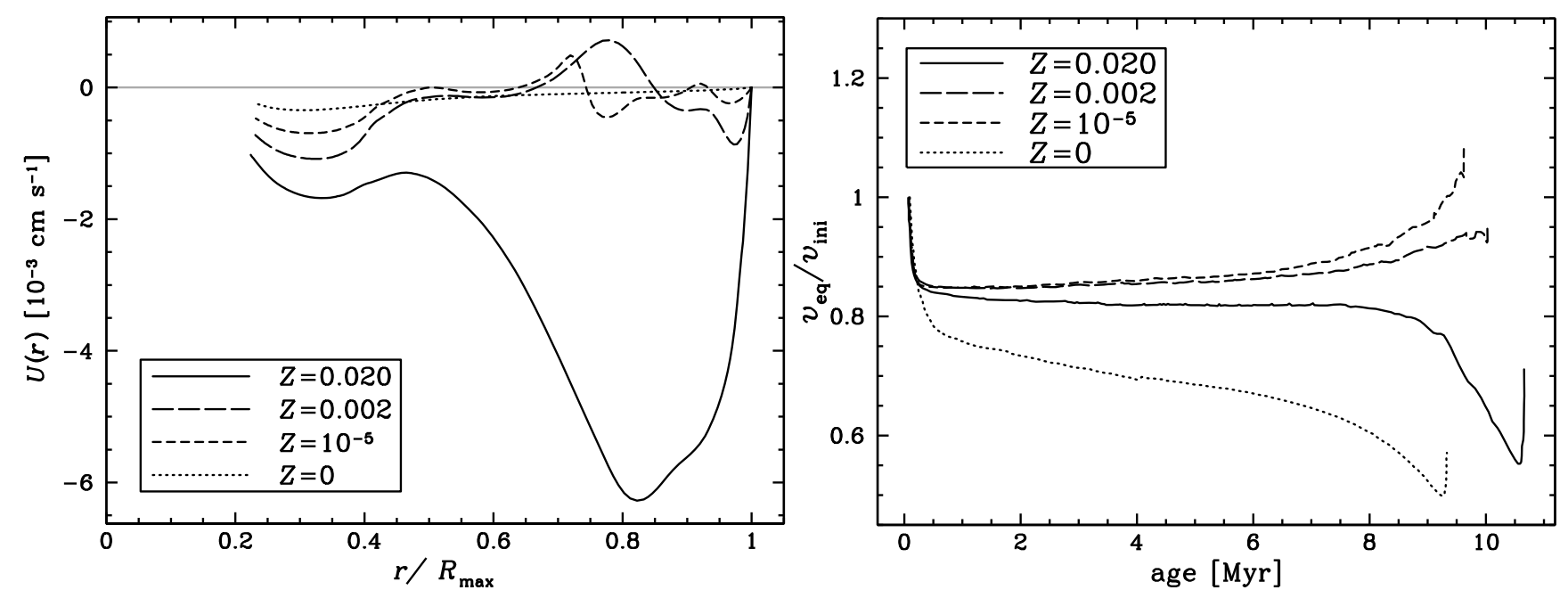

Fig. 3. Models of $20 M_{\odot}$ at various metallicities, with $\Omega_{\text {ini }} / \Omega_{\text {crit }}=0.5$. Left: internal profile of $U(r)$, where $u(r, \theta)$ the vertical component of the velocity of the meridional circulation is $u(r, \theta)=U(r) P_{2}(\cos \theta)$ with $P_{2}$ the second Legendre polynomial. The radius is normalized to the outer one. All the models are at the same evolutionary stage, when the central $\mathrm{H}$ mass fraction is about 0.40 . Right: evolution of the equatorial velocity, normalized to the initial velocity.

earlier in their model (4.4 Myr) than in ours (10 Myr), and in a much more abrupt way (see their Fig. 4). The convective core expands suddenly, the $\mathrm{H}$ abundance rises in the core and thus allows a longer lifetime. The difference in the time at which the CNO cycle ignites arises most probably from differences in the reaction rates and in the equation of state used.

In the case of our $15 M_{\odot}$ non-rotating model, we again find a difference of about $10 \%$ to the work of Marigo et al. (2001), consistent with the difference found in the $9 M_{\odot}$ models. Our lifetime is also $12 \%$ shorter than that calculated by Siess et al. (2002) for their (non-rotating) $15 M_{\odot}$ model, although the overshooting parameter they use is the same as ours. The difference arises from the treatment of the light elements $\left({ }^{7} \mathrm{Be},{ }^{7} \mathrm{Li}\right.$, and $\left.{ }^{8} \mathrm{~B}\right)$ : they are followed explicitly by their code, while ours considers them to be in equilibrium.

In order to compare our results with those of Marigo et al. (2003) at higher masses, we computed the evolution of a nonrotating $120 M_{\odot}$ star up to the end of the MS. Once more the MS lifetime of our model is found to be $10 \%$ shorter than the one computed by the above authors.

\subsection{Evolution of the equatorial velocity}

Our models are started on the ZAMS with a flat rotation profile. As described in Meynet \& Maeder (2000), the meridional circulation then establishes very quickly (1-2\% of the MS time) an equilibrium profile of $\Omega(r)$ inside the star with the core spinning faster than the surface.

After this first adjustment phase, the evolution of the surface (equatorial) velocity results from a delicate interplay between the mass loss (which removes angular momentum at the surface) and the meridional circulation (which brings angular momentum from the core to the surface). How does this interplay behave at $Z=0$, and what are the differences with the situation at higher metallicities? To find an answer, we compared four models of $20 M_{\odot}$ with $\Omega_{\text {ini }} / \Omega_{\text {crit }}=0.50$ at various metallicities, computed with the same code as our set of $Z=0$ models $^{4}$. In the left panel of Fig. 3, we show the internal profile of the radial component of the meridional circulation. Let us focus on the outer cell, which

4 These models are taken from Ekström et al. (2008b). transports the angular momentum outward, when $U(r)$ is negative. The amplitude of the meridional circulation is a factor of 6 higher in the standard metallicity model $(Z=0.020)$ than in the $Z=0.002$ one. This factor amounts to 25 when we compare with the $Z=10^{-5}$ model, and reaches 100 with the $Z=0$ one. This illustrates the effect of the Gratton-Öpik term (see Sect. 2.1) in the expression of the meridional circulation velocity $U(r)$ : when the metallicity decreases, stars are more compact, so the density increases, and thus $U(r)$ decreases. In the right panel of Fig. 3, we see the resulting evolution of the equatorial velocity. At standard metallicity, although the amplitude of the meridional circulation is large, the loss of angular momentum through the radiative winds has the strongest effect, and the equatorial velocity slows down. At low or very low $Z$, the meridional circulation is weak, but the mass loss is so diminished that the models are spinning up. In the case of $Z=0$, there is no mass loss to remove mass and angular momentum, but the meridional circulation is so weak that the evolution of $\Omega(r)$ is very close to local angular momentum conservation, $\Omega r^{2}=$ const.; because of the natural inflation of the external radius, the surface of the model has to slow down.

The behaviour described above is valid for all the models with $M_{\text {ini }}<40 M_{\odot}$ : they are slowing down during the MS evolution, except at the very end of it when they contract briefly before central $\mathrm{H}$ exhaustion. Above $40 M_{\odot}$, the models are spinning up on the MS. This is not surprising, since the meridional circulation is higher in more massive stars (which have a less dense envelope), allowing the transport of angular momentum that is lacking in lower mass models.

During evolution, because of the inflation of the radius, the value of the critical velocity decreases. So even a decelerating model may reach the critical velocity during its evolution. This is indeed what happens with our models. In Fig. 4 we present the evolution of the $\Omega / \Omega_{\text {crit }}$ ratio during the MS. As mentioned in Sect. 2.1, the $9 M_{\odot}$ model is the only one that does not reach the critical limit. For the other models, the higher the mass the sooner the critical limit is attained. The last column of Table 1 gives the central mass fraction of hydrogen when each model reaches the critical velocity.

Once at the critical limit, all the models remain at the limit. In Fig. 4, the 85 and especially the $200 M_{\odot}$ models seem to 
Table 3. Total mass lost and surface abundances of $\mathrm{C}, \mathrm{N}$ and $\mathrm{O}$ at the end of MS phase and at the end of the evolution for the models that undergo mass loss and surface enrichment. Masses are in $M_{\odot}$, velocities in $\mathrm{km} \mathrm{s}^{-1}$ and abundances in mass fraction. The $\mathrm{C}$ abundance is the sum of ${ }^{12} \mathrm{C}$ and ${ }^{13} \mathrm{C}$, the $\mathrm{N}$ abundance is the abundance in ${ }^{14} \mathrm{~N}$ and the $\mathrm{O}$ abundance is the sum of ${ }^{16} \mathrm{O},{ }^{17} \mathrm{O}$ and ${ }^{18} \mathrm{O}$.

\begin{tabular}{|c|c|c|c|c|c|c|c|c|c|c|c|}
\hline \multirow[t]{2}{*}{$\overline{\overline{M_{\text {ini }}}}$} & \multirow[t]{2}{*}{$v_{\text {ini }}$} & \multicolumn{5}{|c|}{$\overline{\text { End of MS }}$} & \multicolumn{5}{|c|}{ End of evolution } \\
\hline & & $M_{\text {lost }}$ & $\mathrm{He}$ & $\mathrm{C}$ & $\mathrm{N}$ & $\mathrm{O}$ & $M_{\text {lost }}$ & $\mathrm{He}$ & $\mathrm{C}$ & $\mathrm{N}$ & $\mathrm{O}$ \\
\hline$\overline{9}$ & 500 & - & 0.2426 & $4.60 e-23$ & $5.91 \mathrm{e}-21$ & $2.35 e-22$ & - & 0.4279 & $5.50 \mathrm{e}-17$ & $5.61 \mathrm{e}-15$ & $1.85 e-16$ \\
\hline 15 & 800 & 0.0306 & 0.2503 & $1.74 \mathrm{e}-14$ & $1.81 \mathrm{e}-12$ & $5.90 \mathrm{e}-14$ & 0.0418 & 0.2646 & $6.36 \mathrm{e}-09$ & $1.83 \mathrm{e}-07$ & $3.38 \mathrm{e}-09$ \\
\hline 25 & 800 & 0.2252 & 0.2517 & $4.34 \mathrm{e}-13$ & $4.02 \mathrm{e}-11$ & $1.20 \mathrm{e}-12$ & 0.2513 & 0.2615 & $3.53 \mathrm{e}-10$ & $9.37 \mathrm{e}-09$ & $1.86 \mathrm{e}-10$ \\
\hline 40 & 800 & 1.0070 & 0.2654 & $2.24 \mathrm{e}-12$ & $1.90 \mathrm{e}-10$ & $5.18 \mathrm{e}-12$ & 2.0074 & 0.3045 & $5.17 \mathrm{e}-07$ & $1.48 \mathrm{e}-05$ & $6.16 \mathrm{e}-07$ \\
\hline 60 & 0 & - & 0.2400 & $0.00 \mathrm{e}+00$ & $0.00 \mathrm{e}+00$ & $0.00 \mathrm{e}+00$ & 0.0001 & 0.2400 & $0.00 \mathrm{e}+00$ & $0.00 \mathrm{e}+00$ & $0.00 \mathrm{e}+00$ \\
\hline 60 & 800 & 2.3200 & 0.2906 & $6.84 \mathrm{e}-12$ & $5.44 \mathrm{e}-10$ & $1.39 \mathrm{e}-11$ & 2.4061 & 0.5328 & $1.23 \mathrm{e}-05$ & $3.54 \mathrm{e}-04$ & $3.34 \mathrm{e}-05$ \\
\hline 85 & 0 & 0.0046 & 0.2400 & $0.00 \mathrm{e}+00$ & $0.00 \mathrm{e}+00$ & $0.00 \mathrm{e}+00$ & 0.0681 & 0.4032 & $6.40 \mathrm{e}-04$ & $3.88 \mathrm{e}-03$ & $1.33 e-04$ \\
\hline 85 & 800 & 4.4363 & 0.3362 & $1.74 \mathrm{e}-11$ & $1.31 \mathrm{e}-09$ & $3.19 \mathrm{e}-11$ & 10.4239 & 0.6141 & $7.29 \mathrm{e}-06$ & $1.81 \mathrm{e}-04$ & $4.14 \mathrm{e}-05$ \\
\hline 200 & 0 & 0.0350 & 0.2400 & $0.00 \mathrm{e}+00$ & $0.00 \mathrm{e}+00$ & $0.00 \mathrm{e}+00$ & 0.0509 & 0.2423 & $7.93 e-13$ & $6.12 \mathrm{e}-11$ & $1.59 \mathrm{e}-12$ \\
\hline 200 & 800 & 16.4426 & 0.5183 & $8.82 \mathrm{e}-11$ & $5.94 \mathrm{e}-09$ & $1.23 \mathrm{e}-10$ & 20.7609 & 0.6685 & $7.53 e-05$ & $2.09 \mathrm{e}-04$ & $1.02 \mathrm{e}-04$ \\
\hline
\end{tabular}

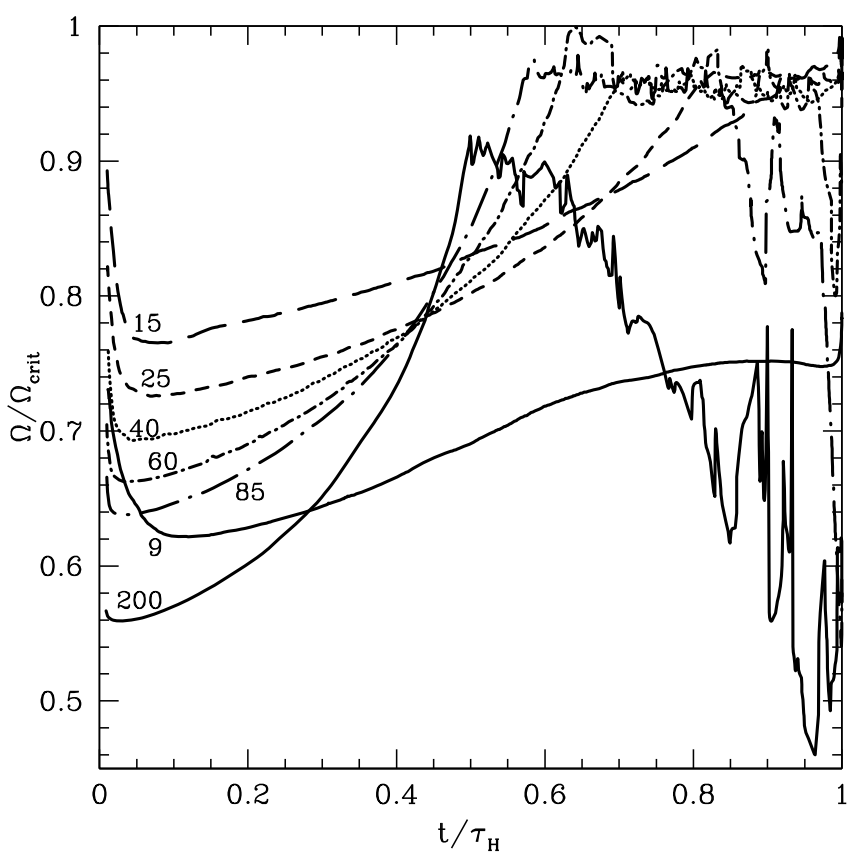

Fig. 4. Evolution of the $\Omega / \Omega_{\text {crit }}$ ratio during the MS. All the models start the MS with $v_{\text {eq }}=800 \mathrm{~km} \mathrm{~s}^{-1}$, except the $9 M_{\odot}$ which starts the MS at $500 \mathrm{~km} \mathrm{~s}^{-1}$.

depart from $\Omega / \Omega_{\text {crit }}=1$, but this is because the limit shown here is only the $\Omega$-limit, where the centrifugal force alone is taken into account to counterbalance the gravity. In the two above models however, the radiative acceleration is strong and they reach the so-called $\Omega \Gamma$-limit, that is the second root of the equation giving the critical velocity: $\boldsymbol{g}_{\text {eff }}[1-\Gamma]=0$ (see Maeder \& Meynet 2000a, for the detailed derivation). The true critical velocity is lowered by the radiative acceleration, and though the plotted $\Omega / \Omega_{\text {crit }}$ ratio becomes lower than 1 , these models are actually at the critical limit and remain at this limit until the end of the MS. At central $\mathrm{H}$ exhaustion, the moderate inflation of the radius brings the surface back to sub-critical velocities.

\subsection{Mass loss and surface abundances}

In Meynet et al. (2006), we show how rotating low-metallicity stars may lose more mass than expected mainly through two processes: first the reaching of the break-up velocity, which leads to a mechanical mass loss of moderate amplitude through a decretion disc; second the enrichment of the surface because of rotational mixing, which leads to a very large enhancement of the radiative mass loss. Now what happens if the metallicity is as low as zero? The total mass lost at the end of MS remains modest in the present models (Table 3): between $0.03 M_{\odot}$ for the $15 M_{\odot}$ model (which reaches the critical limit only at the very end of the MS, when $X_{\mathrm{c}}=0.02$ ), and $16.4 M_{\odot}$ for the $200 M_{\odot}$ model (which reaches the break-up limit at $X_{\mathrm{c}}=0.43$ ). The mechanical mass loss removes only the outermost layers, which have a very low density, so only a little mass is removed during this phase.

After the MS, as mentioned in Sect. 3.1, the models remain in the blue part of the HRD most of the $\mathrm{CHeB}$ phase and then their redward incursion is moderate. The outer convective zone that develops remains very thin, so the dredge-up does not dig deeply enough (see the Kippenhahn diagrams in Fig. 5). Most of the post-MS evolution occurs with a surface metallicity much lower than $10^{-6}$. As a result, the mass lost during $\mathrm{CHeB}$ remains very low: it amounts to at most $6 M_{\odot}$ in the case of the $85 M_{\odot}$ model, which is the model that loses the most mass at this stage.

The total mass lost by the models during the whole evolution is small. The $200 M_{\odot}$ model loses $20.7 M_{\odot}$, the $85 M_{\odot}$ model $10.4 M_{\odot}$. The models have to be as massive as $40 M_{\odot}$ or above to lose more than $1 M_{\odot}$. This picture is very different to the evolution with just a small fraction of metals: the $60 M_{\odot}$ at $Z=10^{-8}$ presented in Meynet et al. (2006) loses $36 M_{\odot}$, among which $27 M_{\odot}$ are due to the enhancement of the radiative winds by the surface self-enrichment. At $Z=0$, a similar model loses $2.4 M_{\odot}$ only. To check if the choice of a higher initial equatorial velocity could significantly enhance the mass loss in $Z=0$ models, we have computed a test model of $40 M_{\odot}$ with $v_{\text {ini }}=900 \mathrm{~km} \mathrm{~s}^{-1}$ (i.e. $\Omega / \Omega_{\text {crit }}=0.83$ ). We evolved it until the end of CHeB. During the MS evolution, it reaches the critical limit when its central $\mathrm{H}$ mass fraction is $X_{\mathrm{c}}=0.43$ and loses $1.702 M_{\odot}$ until the end of the MS. The total mass lost at the end of $\mathrm{CHeB}$ amounts to $1.734 M_{\odot}$ which compares well with the $1.571 M_{\odot}$ lost by the model with a slower $v_{\text {ini }}$ at the same stage. We can thus conclude that the choice of a higher $v_{\text {ini }}$ is not determinant in altering mass loss.

The non-rotating models do not lose mass at all: even the $200 M_{\odot}$ model loses only a few hundredths of a solar mass. We will see in Sect. 4.3 that some of our models may be able to contribute to the chemical enrichment of the medium only through their wind ejecta. In that context, while the non-rotating models do not contribute at all, the rotating ones will leave a modest imprint on their surroundings.

We cannot directly compare our results with the literature: the only study that does not compute the evolution at constant mass is the one by Marigo et al. (2003) and their masses do 

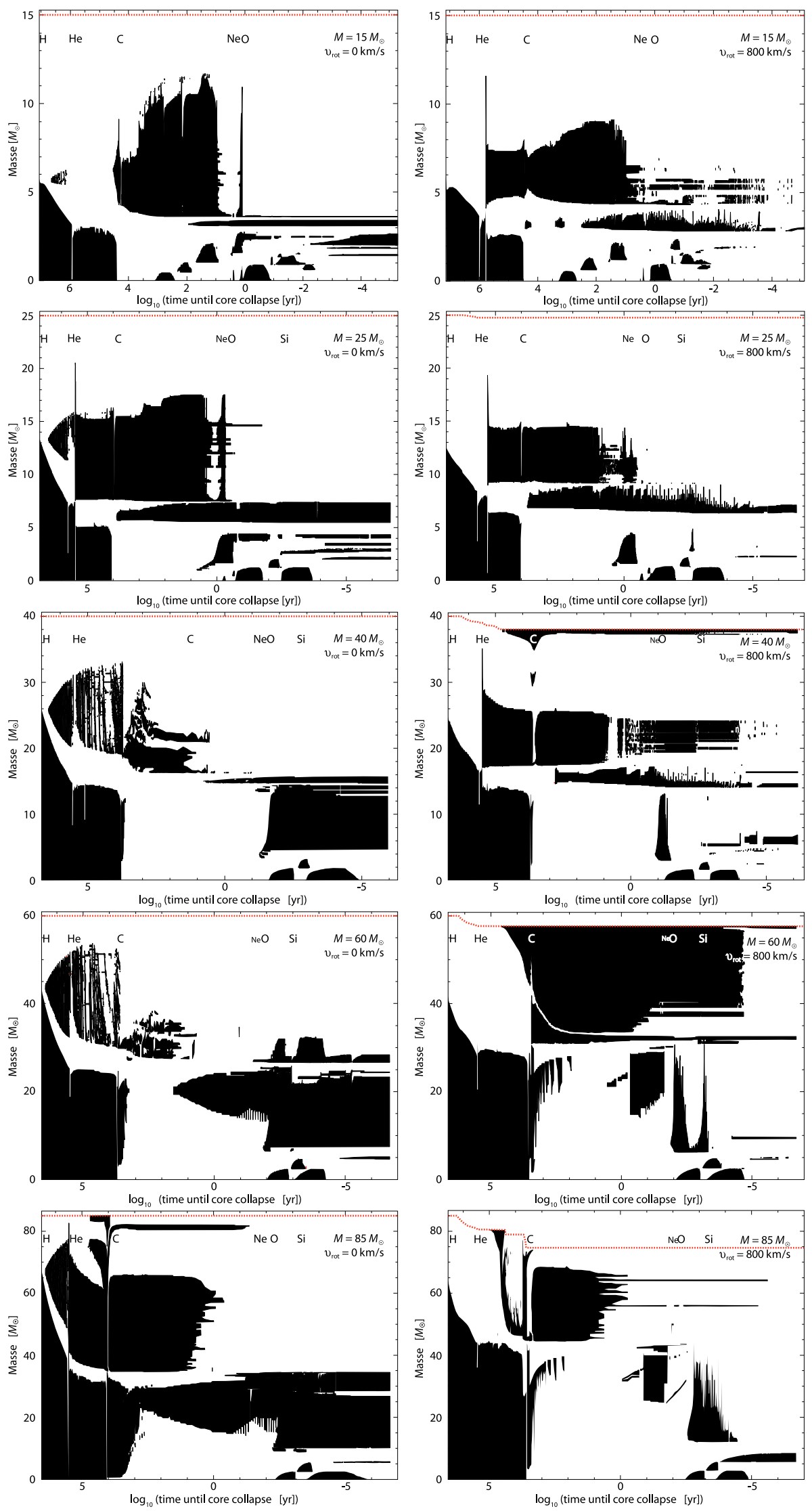

Fig. 5. Kippenhahn diagrams (evolution of the structure) for the models between 15 and $85 M_{\odot}$. Left column: without rotation; right column: with rotation. The black areas show the convective zones. The (red) dotted line at the top of the figures is the total mass of the model. The on-set of the different burning phases is indicated at the top of each window. 
not match ours exactly. Nevertheless, we can make a qualitative comparison. Our rotating $200 M_{\odot}$ model experiences a much stronger mass loss than what they found for their rotating $250 M_{\odot}$ model, which loses $3.35 M_{\odot}$ only, that is 6 times less than our slightly less heavy model. Their models have been computed with a lower initial velocity, and despite the maximal coupling brought by the rigid body rotation, they attain the critical limit only at the very end of the MS phase. The mass loss they adopt then $\left(M_{\text {crit }}=10^{-3} M_{\odot} \mathrm{yr}^{-1}\right)$ is about 2 orders of magnitude higher than that resulting from our treatment of the critical mass loss, but the period during which they apply it is short.

\section{Final structure and light element yields}

\subsection{Kippenhahn diagrams}

In Fig. 5 we present the Kippenhahn diagrams (evolution of the structure) of the models between 15 and $85 M_{\odot}$. Generally, rotation suppresses the convective instability outside the H-burning core by smoothing the abundance gradient of helium above the core. The CNO shell boost is clearly visible in the diagrams of the non-rotating $25 M_{\odot}$ and the rotating 15,25 and $40 M_{\odot}$ models: the H-burning shell suddenly becomes convective and the core is abruptly reduced. We can discover in the rotating $60 M_{\odot}$ diagram a similar boost, but only at the beginning of shell $\mathrm{He}$ burning. This boost left no signature in the HRD.

As mentioned above, the rotating 15 and $25 M_{\odot}$ models remain in the blue part of the HRD and do not develop any outer convective zone $(\mathrm{OCZ})$. The rotating $40 M_{\odot}$ presents a very thin one and the $85 M_{\odot}$ a very early and short-lived one. On the contrary, the $60 M_{\odot}$ develops a deeper $\mathrm{OCZ}$ at the end of $\mathrm{CHeB}$ and for almost the rest of its evolution until the pre-SN stage. Its surface enrichment is thus larger than that presented by the $85 M_{\odot}$ model. The only non-rotating model that develops an OCZ is the $85 M_{\odot}$. Although this convective zone disappears quickly, it suffices to dredge up heavy elements brought near the surface by the CNO shell boost, and it is the model that shows the highest level of enrichment at the end of its evolution (see Table 3).

Limongi et al. (2000) show that at standard metallicity, the transition from convective to radiative core $\mathrm{C}$ burning occurs between 15 and $20 M_{\odot}$. From Fig. 1 of Limongi et al. (2001), we deduce that at $Z=0$, this transition occurs at slightly higher masses, between 20 and $25 M_{\odot}$. We note that our $15 M_{\odot}$ models have convective $\mathrm{C}$-burning cores, while cores are radiative in the case of our $25 M_{\odot}$ models, in agreement with Limongi et al. (2001). In the non-rotating models with $M \geq 40 M_{\odot}$, the shell $\mathrm{C}$ burning is convective up to the end of the hydrostatic evolution, while in the rotating models, the shell experiences a few flashes but otherwise remains radiative.

\subsection{Stellar yields}

The yields calculated from stellar models are strongly correlated to the value of the CO-core mass, $M_{\mathrm{CO}}$. We give the values of $M_{\mathrm{CO}}$ of our models in Table 2. They were determined as in Heger et al. (2000a) as the mass coordinate at which the abundance of He drops below $10^{-3}$ (in mass fraction). From these values, we determined the remnant masses of our models, $M_{\text {rem }}$, with the relation between $M_{\text {rem }}$ and $M_{\mathrm{CO}}$ described in Maeder (1992)

In Table 4 we present the total stellar yields for the light elements that are not significantly modified by the explosion (Limongi \& Chieffi 2002). The total yields are the sum of the pre-SN yields $\left(m p_{\mathrm{im}}^{\mathrm{preSN}}\right)$ and the wind yields $\left(m p_{\mathrm{im}}^{\text {winds }}\right)$. The

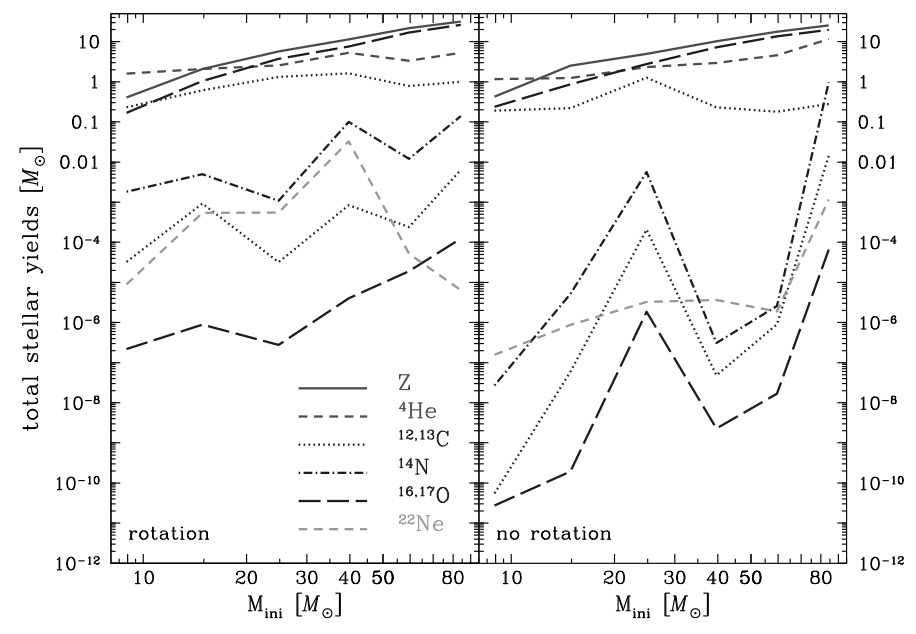

Fig. 6. Total stellar yields as a function of initial mass for our $Z=0$ models. Left: models with rotation; right: non-rotating models.

pre-SN contribution from a star of initial mass $m$ to the stellar yield of an element $i$ is:

$m p_{\mathrm{im}}^{\mathrm{preSN}}=\int_{M_{\mathrm{rem}}}^{M_{\mathrm{fin}}}\left[X_{i}\left(m_{r}\right)-X_{i}^{0}\right] \mathrm{d} m_{r}$

with $X_{i}^{0}$ the initial abundance (in mass fraction) of element $i$ $\left(X_{i}^{0}=0\right.$ for all elements except $\left.\mathrm{He}\right)$, and $X_{i}\left(m_{r}\right)$ its final abundance at the Lagrangian coordinate $m_{r}$. The winds contribution is:

$m p_{\mathrm{im}}^{\text {winds }}=\int_{0}^{\tau(m)} \dot{M}(m, t)\left[X_{i}^{\mathrm{S}}(m, t)-X_{i}^{0}\right] \mathrm{d} t$

where $\tau(m)$ is the final age of the star with initial mass $m, \dot{M}(m, t)$ the mass loss rate when the age of the star is $t$, and $X_{i}^{\mathrm{S}}(m, t)$ the surface abundance (in mass fraction) of element $i$ at age $t$. Most of the time, the total stellar yields are dominated by the pre-SN contribution.

We did not include the $200 M_{\odot}$ models in Table 4 because they have not undergone the shell He-burning phase, which noticeably modifies the results. The $9 M_{\odot}$ models were also stopped before central $\mathrm{C}$ burning, but as we mentioned, the core was strongly degenerate at carbon ignition, which could lead to the complete destruction of the star without further processing. Note that the carbon ignition occurred at the edge of the core, so there is a possibility that the star survived $\mathrm{C}$ burning. In that case, since the mass included below the He-burning shell is less than the limiting mass for $\mathrm{Ne}$ burning $\left(1.37 M_{\odot}\right)$, the star is expected to collapse in a type II SN by electron capture on a degenerate O-Ne-Mg core (Nomoto 1984). This would modify the pre-SN yields presented here since the $\mathrm{C}$ burning would have processed the matter of the core. We would thus expect a reduction of ${ }^{12} \mathrm{C}$ and an enhancement of ${ }^{13} \mathrm{C},{ }^{14} \mathrm{~N},{ }^{16} \mathrm{O}$ and ${ }^{22} \mathrm{Ne}$. In the case of the $15 M_{\odot}$ models, the evolution was stopped at the end of central $\mathrm{O}$ burning. At that time, the mass interior to the C-burning shell is higher than the Chandrasekhar mass, so the star is expected to undergo an off-centre $\mathrm{Si}$ flash and to end with an Fe core (Nomoto 1984). We then expect the yields presented here to be modified slightly (reduction of $\mathrm{C}, \mathrm{N}$ and $\mathrm{Ne}$, enhancement of $\mathrm{O}$ ). We have checked the amplitude of the variation when the yields are calculated at the end of $\mathrm{O}$ burning or at the end of Si burning in the models that have been followed until the pre-SN phase, so we do not expect the yields of the $15 M_{\odot}$ models given in Table 4 to vary by more than $15 \%$. 
Table 4. Total stellar yields at the pre-supernova stage, in $M_{\odot}$. In parenthesis, the "wind-only" contribution is given for the models for which no SN contribution is expected (see Sect. 4.3). The masses of the models are in $M_{\odot}$ and the velocities in $\mathrm{km} \mathrm{s}^{-1}$.

\begin{tabular}{|c|c|c|c|c|c|c|c|c|c|c|c|}
\hline$M_{\text {ini }}$ & $v_{\text {ini }}$ & $\frac{{ }^{3} \mathrm{He}}{10^{-4}}$ & ${ }^{4} \mathrm{He}$ & ${ }^{12} \mathrm{C}$ & ${ }^{13} \mathrm{C}$ & ${ }^{14} \mathrm{~N}$ & ${ }^{16} \mathrm{O}$ & ${ }^{17} \mathrm{O}$ & ${ }^{18} \mathrm{O}$ & ${ }^{22} \mathrm{Ne}$ & $Z$ \\
\hline 9 & 0 & -0.53 & 1.17 & 0.19 & $5.54 \mathrm{e}-11$ & $2.68 \mathrm{e}-8$ & 0.24 & $2.74 \mathrm{e}-11$ & $4.45 e-9$ & $1.58 \mathrm{e}-7$ & 0.43 \\
\hline 9 & 500 & -0.65 & 1.59 & 0.24 & $3.27 e-5$ & $1.81 \mathrm{e}-3$ & 0.17 & $2.20 \mathrm{e}-7$ & $8.20 \mathrm{e}-6$ & $8.98 \mathrm{e}-6$ & 0.41 \\
\hline 15 & 0 & -1.02 & 1.24 & 0.22 & $5.94 \mathrm{e}-8$ & $5.13 e-6$ & 0.86 & $1.93 \mathrm{e}-10$ & $2.68 \mathrm{e}-9$ & $8.73 \mathrm{e}-7$ & 2.53 \\
\hline 15 & 800 & -1.23 & 2.10 & 0.61 & $9.21 \mathrm{e}-4$ & $4.99 \mathrm{e}-3$ & 1.05 & $8.88 \mathrm{e}-7$ & $7.17 \mathrm{e}-6$ & $5.44 \mathrm{e}-4$ & 2.10 \\
\hline 25 & 0 & -1.97 & 2.32 & 1.26 & $2.09 \mathrm{e}-4$ & $5.69 e-3$ & 2.78 & $1.86 \mathrm{e}-6$ & $1.79 \mathrm{e}-9$ & $3.27 \mathrm{e}-6$ & 4.93 \\
\hline 25 & 800 & $\begin{array}{l}-2.13 \\
(-0.02)\end{array}$ & $\begin{array}{c}2.57 \\
(0.003)\end{array}$ & $\begin{array}{c}1.32 \\
(5 e-14)\end{array}$ & $\begin{array}{c}3.21 \mathrm{e}-5 \\
(1.59 \mathrm{e}-14)\end{array}$ & $\begin{array}{c}1.08 \mathrm{e}-3 \\
(6.86 \mathrm{e}-12)\end{array}$ & $\begin{array}{c}3.76 \\
(2 \mathrm{e}-13)\end{array}$ & $\begin{array}{c}2.75 \mathrm{e}-7 \\
(1.51 \mathrm{e}-15)\end{array}$ & $\begin{array}{c}7.78 \mathrm{e}-6 \\
(6.51 \mathrm{e}-19)\end{array}$ & $\begin{array}{c}5.54 \mathrm{e}-4 \\
(1.01 \mathrm{e}-26)\end{array}$ & $\begin{array}{c}5.75 \\
(7 e-12)\end{array}$ \\
\hline 40 & 0 & $\begin{array}{l}-3.21 \\
(0.00)\end{array}$ & $\begin{array}{c}2.94 \\
(0.00)\end{array}$ & $\begin{array}{c}0.23 \\
(0.00)\end{array}$ & $\begin{array}{c}4.85 \mathrm{e}-8 \\
(0.00)\end{array}$ & $\begin{array}{c}3.09 \mathrm{e}-7 \\
(0.00)\end{array}$ & $\begin{array}{c}7.25 \\
(0.00)\end{array}$ & $\begin{array}{c}2.29 \mathrm{e}-9 \\
(0.00)\end{array}$ & $\begin{array}{c}1.55 \mathrm{e}-8 \\
(0.00)\end{array}$ & $\begin{array}{c}3.63 \mathrm{e}-6 \\
(0.00)\end{array}$ & $\begin{array}{c}10.2 \\
(0.00)\end{array}$ \\
\hline 40 & 800 & $\begin{array}{l}-3.32 \\
(-0.17)\end{array}$ & $\begin{array}{c}5.32 \\
(0.05)\end{array}$ & $\begin{array}{c}1.63 \\
(3 e-8)\end{array}$ & $\begin{array}{c}8.41 \mathrm{e}-4 \\
(7.96 \mathrm{e}-9)\end{array}$ & $\begin{array}{c}1.00 \mathrm{e}-1 \\
(1.07 \mathrm{e}-6)\end{array}$ & $\begin{array}{c}7.57 \\
(4 e-08)\end{array}$ & $\begin{array}{c}4.04 \mathrm{e}-6 \\
(5.89 \mathrm{e}-11)\end{array}$ & $\begin{array}{c}6.74 \mathrm{e}-5 \\
(5.43 \mathrm{e}-14)\end{array}$ & $\begin{array}{c}3.30 \mathrm{e}-2 \\
(2.64 \mathrm{e}-11)\end{array}$ & $\begin{array}{c}11.4 \\
(1 \mathrm{e}-6)\end{array}$ \\
\hline 60 & 0 & $\begin{array}{l}-4.87 \\
(0.00)\end{array}$ & $\begin{array}{c}4.58 \\
(0.00)\end{array}$ & $\begin{array}{c}0.18 \\
(0.00)\end{array}$ & $\begin{array}{c}9.07 \mathrm{e}-7 \\
(0.00)\end{array}$ & $\begin{array}{c}2.58 \mathrm{e}-6 \\
(0.00)\end{array}$ & $\begin{array}{c}13.6 \\
(0.00)\end{array}$ & $\begin{array}{c}1.69 \mathrm{e}-8 \\
(0.00)\end{array}$ & $\begin{array}{c}2.26 \mathrm{e}-7 \\
(0.00)\end{array}$ & $\begin{array}{c}1.89 \mathrm{e}-6 \\
(0.00)\end{array}$ & $\begin{array}{c}17.7 \\
(0.00)\end{array}$ \\
\hline 60 & 800 & $\begin{array}{l}-4.76 \\
(-0.21)\end{array}$ & $\begin{array}{c}3.33 \\
(0.04)\end{array}$ & $\begin{array}{c}0.79 \\
(2 \mathrm{e}-8)\end{array}$ & $\begin{array}{c}2.39 \mathrm{e}-4 \\
(7.10 \mathrm{e}-9)\end{array}$ & $\begin{array}{c}1.20 \mathrm{e}-2 \\
(9.00 \mathrm{e}-7)\end{array}$ & $\begin{array}{c}16.9 \\
(9 \mathrm{e}-8)\end{array}$ & $\begin{array}{c}1.89 \mathrm{e}-5 \\
(1.94 \mathrm{e}-10)\end{array}$ & $\begin{array}{c}1.33 \mathrm{e}-4 \\
(8.18 \mathrm{e}-14)\end{array}$ & $\begin{array}{c}5.24 \mathrm{e}-5 \\
(4.12 \mathrm{e}-11)\end{array}$ & $\begin{array}{c}21.7 \\
(1 \mathrm{e}-6)\end{array}$ \\
\hline 85 & 0 & $\begin{array}{c}-7.22 \\
(-0.004)\end{array}$ & $\begin{array}{l}11.70 \\
(0.01)\end{array}$ & $\begin{array}{c}0.28 \\
(3 e-5)\end{array}$ & $\begin{array}{c}1.46 \mathrm{e}-2 \\
(8.20 \mathrm{e}-6)\end{array}$ & $\begin{array}{c}9.87 \mathrm{e}-1 \\
(2.42 \mathrm{e}-4)\end{array}$ & $\begin{array}{c}19.7 \\
(8 e-6)\end{array}$ & $\begin{array}{c}6.63 e-5 \\
(7.26 e-9)\end{array}$ & $\begin{array}{c}1.17 \mathrm{e}-7 \\
(1.50 \mathrm{e}-11)\end{array}$ & $\begin{array}{c}1.18 \mathrm{e}-3 \\
(3.99 \mathrm{e}-7)\end{array}$ & $\begin{array}{c}25.5 \\
(3 e-4)\end{array}$ \\
\hline 85 & 800 & -6.15 & 5.31 & 1.00 & $6.44 \mathrm{e}-3$ & $1.40 \mathrm{e}-1$ & 26.2 & $1.24 \mathrm{e}-4$ & $2.32 \mathrm{e}-7$ & $6.47 e-6$ & 31.6 \\
\hline
\end{tabular}

Rotation leads to larger yields for almost all the isotopes and for almost all the masses (see Fig. 6). The exceptions are the non-rotating models that undergo a strong $\mathrm{CNO}$ shell boost: the 25 and $85 M_{\odot}$ models, which produce larger amounts of ${ }^{13} \mathrm{C}$ and ${ }^{14} \mathrm{~N}$. The $25 M_{\odot}$ model overtakes its rotating counterpart also in the production of ${ }^{17} \mathrm{O}$, and the $85 M_{\odot}$ model produces more ${ }^{4} \mathrm{He}$ and ${ }^{22} \mathrm{Ne}$ than the rotating one. Otherwise, the enhancement of the yields brought by the rotational mixing may reach orders of magnitude, especially so for the production of primary ${ }^{14} \mathrm{~N}$ : a factor of $10^{5}$ between the rotating and non-rotating $9 M_{\odot}$ models and even a factor of $10^{6}$ in the case of the $40 M_{\odot}$ models. We note that rotation makes the production of ${ }^{14} \mathrm{~N}$ robust through the whole mass range, while there are enormous differences from mass to mass in the non-rotating models.

Figure 7 shows a comparison of the $\mathrm{CNO}$ yields of all our $Z=0$ models with the yields of the non-rotating models at $Z=0$ from Chieffi \& Limongi (2004) and those of the rotating models at $Z=10^{-8}$ from Hirschi (2007). For ${ }^{12} \mathrm{C}$ (left) and ${ }^{16} \mathrm{O}$ (right), our non-rotating 15 and $25 M_{\odot}$ yields compare within a factor of 2 or 3 with those of Chieffi \& Limongi (2004). Their $25 M_{\odot}$ produces a huge amount of ${ }^{14} \mathrm{~N}$ (centre), even a factor $\sim 10$ higher than ours, so the CNO boost of the H-burning shell seems to be a robust feature in this mass domain. Compared to the ${ }^{12} \mathrm{C}$ produced at $Z=10^{-8}$, the production of our rotating 60 and $85 M_{\odot}$ models is smaller, while it is almost equal or larger for the 9 to $40 M_{\odot}$ models. This can be understood because our high mass models do not undergo a shell boost, so their He-burning core is larger, which leads to a lower ${ }^{12} \mathrm{C}$ yield. The ${ }^{16} \mathrm{O}$ production is about the same at $Z=10^{-8}$ and $Z=0$. Our PopIII models have slightly larger ${ }^{16} \mathrm{O}$ yields than the models of Hirschi (2007), but we use a higher overshooting parameter which leads to larger $\mathrm{CO}$ cores and thus greater ${ }^{16} \mathrm{O}$ production.

An interesting point is the production of primary nitrogen at very low metallicity. Meynet \& Maeder (2002) show that this production increases when the metallicity decreases, and Hirschi (2007) confirms the trend. We should thus expect a still higher production at $Z=0$, but this is not the case. ${ }^{14} \mathrm{~N}$ is produced systematically less at $Z=0$ than at $Z=10^{-8}$. We relate this change of behaviour to the fact that the $Z=0$ stars burn their hydrogen at temperatures hot enough to burn some He as well and do not evolve into the red part of the HR diagram (or do so very slowly during $\mathrm{CHeB}$ ) as can be seen in Fig. 1 (left). Due to this fact, the stars undergo much less mixing during the $\mathrm{CHeB}$ phase. Indeed, no redwards evolution means that the gradient of the angular velocity at the border of the He-burning core remains much shallower and triggers much less mixing. On the contrary, when a small amount of metal is present, stars begin to burn their hydrogen through the CNO cycle from the start. Thus the central temperatures during the MS phases are well below those required for He-burning. At the end of the MS phase, the core contracts and the stars evolve quickly to the red. A steep angular velocity gradient appears at the core border and triggers strong mixing. Figure 7 shows that there is even a difference of almost two orders of magnitude between the production of the $20 M_{\odot}$ model at $Z=10^{-8}$ and the $25 M_{\odot}$ model at $Z=0$. This can be explained as follows: the $Z=10^{-8}$ model experiences a second CNO shell boost during shell He burning, which enhances its ${ }^{14} \mathrm{~N}$ production by about two orders of magnitude.

\subsection{Fate of the models}

At high metallicity, massive stars experience very strong mass loss. Their final mass is therefore very low (even smaller than the final mass of stars with an initial mass $M_{\text {ini }} \leq 25 M_{\odot}$, see Maeder 1992). Thus, the fate of all massive stars is a SN explosion, whatever their initial mass.

At very low metallicity, this is no longer true. Heger \& Woosley (2002) and Heger et al. (2003) determine the limiting $M_{\mathrm{He}}$ for which the fate will be:

- a type II SN: $M_{\mathrm{He}}<9 M_{\odot}$;

- a black hole $(\mathrm{BH})$ by fallback: $9 M_{\odot} \leq M_{\mathrm{He}}<15 M_{\odot}$;

- a direct BH: $15 M_{\odot} \leq M_{\mathrm{He}}<40 M_{\odot}$;

- a pulsational pair-instability followed by a SN with BH formation: $40 M_{\odot} \leq M_{\mathrm{He}}<64 M_{\odot}$;

- a pair-instability SN (PISN): $64 M_{\odot} \leq M_{\mathrm{He}} \leq 133 M_{\odot}$;

- or a direct BH collapse: $M_{\mathrm{He}}>133 M_{\odot}$. 


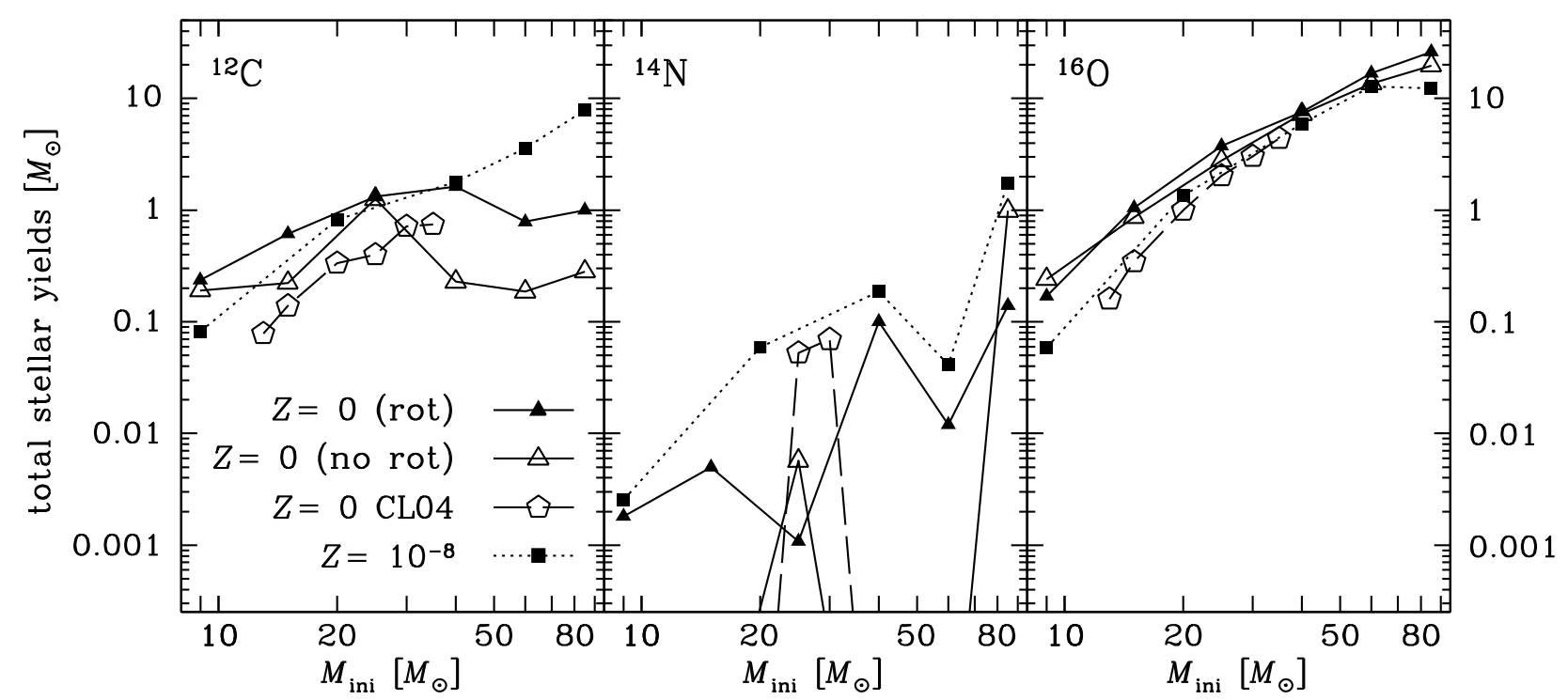

Fig. 7. Yield comparison between the non-rotating $Z=0$ models from Chieffi \& Limongi (2004, CL04, open pentagons), the rotating $Z=$ $10^{-8}$ models from Hirschi (2007, filled squares) and our rotating (filled triangles) and non-rotating (open triangles) $Z=0$ models. Left: ${ }^{12} \mathrm{C}$; centre: ${ }^{14} \mathrm{~N}$; right: ${ }^{16} \mathrm{O}$.

Table 5. Some rotational properties of the iron core of our models: the mass $M_{\mathrm{Fe}}$ where the abundance of the sum ${ }^{48} \mathrm{Cr}+{ }^{52} \mathrm{Fe}+{ }^{56} \mathrm{Ni}$ amounts to $50 \%$ in mass fraction; the angular velocity $\Omega$ taken in the middle of the iron core (the profile of $\Omega(m)$ is almost flat in the core, so this value represents the mean value of $\Omega$ in the iron core); the maximum specific angular momentum $j$ inside the core, corresponding to the first peak seen in Fig. 8 (left); the total angular momentum $\mathcal{L}$ and the parameter $\beta=E_{\mathrm{rot}} /\left|E_{\mathrm{pot}}\right|$, both taken at the border of the iron core.

\begin{tabular}{rccccc}
\hline \hline Mass \\
{$\left[M_{\odot}\right]$} & $\begin{array}{c}M_{\mathrm{Fe}} \\
{\left[M_{\odot}\right]}\end{array}$ & $\begin{array}{c}\Omega \\
{\left[\mathrm{s}^{-1}\right]}\end{array}$ & $\begin{array}{c}j \\
{\left[\mathrm{~cm}^{2} \mathrm{~s}^{-1}\right]}\end{array}$ & $\begin{array}{c}\mathcal{L} \\
{\left[\mathrm{g} \mathrm{cm}^{2} \mathrm{~s}^{-1}\right]}\end{array}$ & $\begin{array}{c}\beta \\
\%\end{array}$ \\
\hline 25 & 1.327 & 0.4232 & $4.073 \mathrm{e}+16$ & $5.837 \mathrm{e}+49$ & 1.5833 \\
40 & 1.555 & 0.3547 & $3.667 \mathrm{e}+16$ & $5.963 \mathrm{e}+49$ & 1.0046 \\
60 & 2.516 & 0.1578 & $4.338 \mathrm{e}+16$ & $1.152 \mathrm{e}+50$ & 0.5449 \\
85 & 2.779 & 0.1062 & $3.913 \mathrm{e}+16$ & $1.182 \mathrm{e}+50$ & 0.3624 \\
\hline
\end{tabular}

Note that the limits above are obtained by non-rotating models, and they may not be appropriate for rotating models, as shown by Fryer et al. (2001). But since we are lacking more accurate determinations, we applied them to all our models. A star that ends its life as a $\mathrm{BH}$ is supposed to contribute to the chemical enrichment of its surrounding only through its winds. We give in parenthesis in Table 4 this "wind-only" contribution for the models that, according to the limits determined above, end their life as BH by fallback or by direct collapse.

The wind yields are very small, since we have seen that the winds are very weak (Sect. 3.4). The $25 M_{\odot}$ model for instance, releases a total metal amount of less than $10^{-7} M_{\odot}$ through its winds while it produces a little more than $5 M_{\odot}$ in the SN scenario. The $60 M_{\odot}$ model contributes to the enrichment of the early Universe with a mere $10^{-5} M_{\odot}$ of metals. Note that the net enrichment depends of course on the amount of metal ejected, but also on the volume in which it is diluted. The wind contribution is expected to be a slow one, so it will be diluted in a smaller volume. The net enrichment may not be much smaller than a SN contribution diluted in a larger volume.

Rotation changes the mass of the He-cores at the end of the evolution. They are usually more massive because of the rotationally-induced mixing, and this may have consequences for the way the star ends its life. While the $25 M_{\odot}$ model without rotation ends as a type II SN, the rotating one is supposed to produce a weak explosion and a $\mathrm{BH}$ by fallback. The non-rotating $85 M_{\odot}$ model remains in the direct $\mathrm{BH}$ domain while the rotating one reaches a He-core mass that implies pulsational pair-instability, followed by an Fe-core collapse into a $\mathrm{BH}$. Its true yields must thus be very close to the pre-SN value.

As mentioned in Sect. 3.1, none of our models meet the conditions for becoming WR stars. They end their evolution having kept their envelope, and thus seem to fail to become a GRB progenitor as defined in the collapsar model of Woosley (1993). However, we have seen that the rotating $85 M_{\odot}$ is likely to undergo pulsational pair instability ${ }^{5}$ and thus lose some mass in this process. If the mass lost is large, it may become a GRB progenitor since the very low core-envelope coupling has maintained a high angular momentum in the core. Fig. 8 (left) shows that in the innermost parts of this model, the specific angular momentum is higher than that needed to create an accretion disc around a BH.

More generally, we expect that rotation will change the way a star collapses. Shimizu et al. (2001) show that an anisotropy of only $10 \%$ in the neutrino flux can boost the energy of the explosion. Walder et al. (2005) and Yamasaki \& Yamada (2005) show that the rotation-induced asphericity of the matter accretion rate is an even more important factor that helps produce a robust explosion. The geometry of the system leads most probably to a jet-like explosion. Table 5 gives the rotational characteristics of the iron core of our models and Fig. 8 (right) the distribution of angular velocity inside the $85 M_{\odot}$ model. All models have kept a high total angular momentum, and meet the conditions to produce a maximally rotating $\mathrm{BH}$. Though detailed calculations would be needed to ascertain this point, the true fate of our models could be much more explosive than previously supposed. In the chemical evolution model discussed below (see Sect. 5), we shall use the total yields, implicitly assuming that the material above $M_{\text {rem }}$ will be ejected.

The centrifugal force has a stabilising effect on the collapsing core, so the core bounce may occur at sub-nuclear densities. This alters the emission of gravitational waves (GW).

\footnotetext{
5 This process has not been followed in the present models.
} 

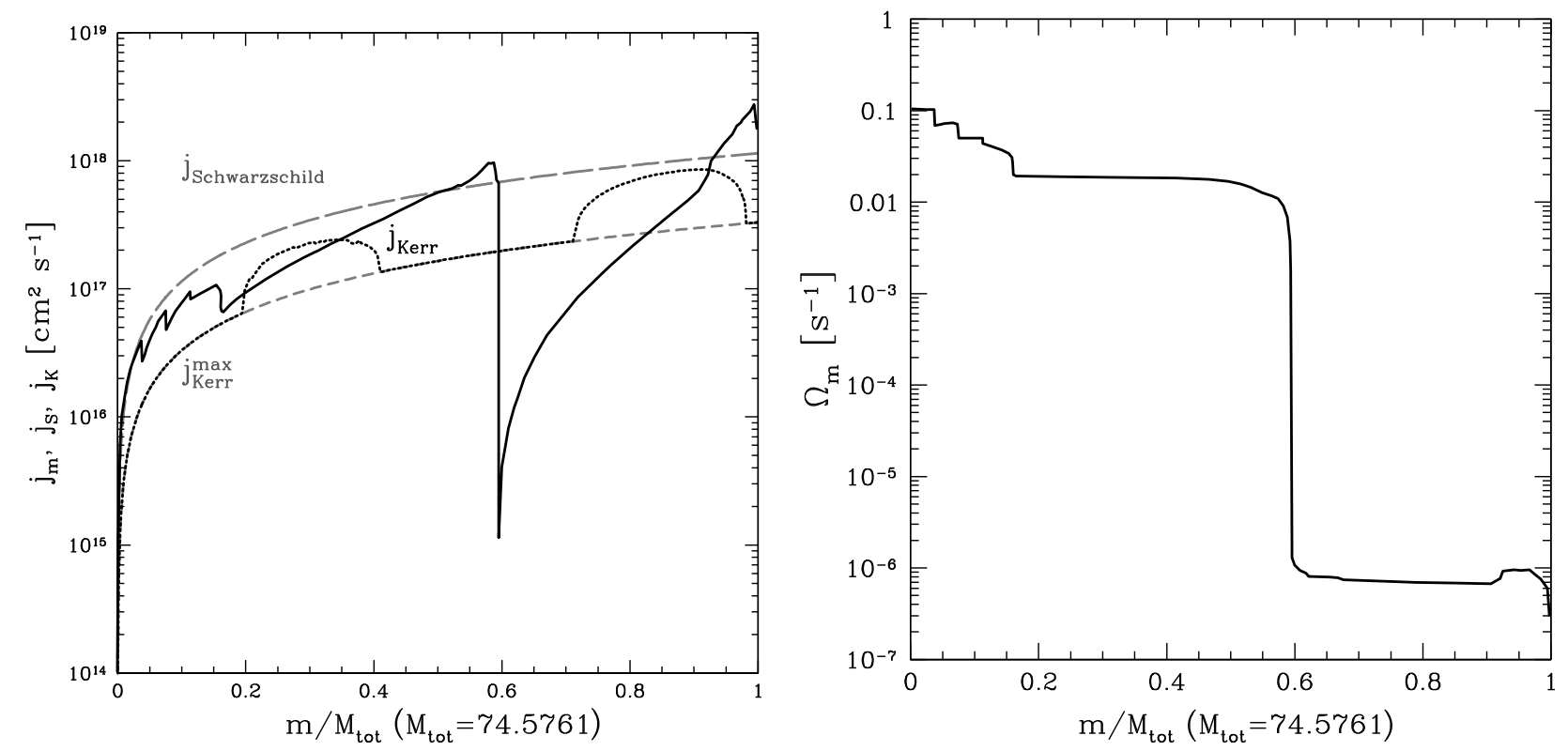

Fig. 8. Left: profile of the specific angular momentum $j_{\mathrm{m}}$ inside our $85 M_{\odot}$ model at the end of hydrostatic core Si-burning (solid line). The dotted line is $j_{\mathrm{K}}=r_{\mathrm{LSO}} c$ (Shapiro \& Teukolsky 1983, p. 428), where the radius of the last stable orbit, $r_{\mathrm{LSO}}$, is given by $r_{\mathrm{ms}}$ in formula (12.7.24) from Shapiro \& Teukolsky $\left(1983\right.$, p. 362) for a circular orbit in the Kerr metric. $j_{\mathrm{K}}$ is the minimum specific angular momentum necessary to form an accretion disc around a rotating black hole. $j_{\text {Schwarschild }}=\sqrt{12} \mathrm{Gm} / \mathrm{c}$ (long-dashed line) and $j_{\mathrm{Kerr}}=G m / c$ (short-dashed line) is the minimum specific angular momentum necessary for a non-rotating and a maximally-rotating black hole, respectively. Right: profile of $\Omega_{\mathrm{m}}$ inside the same model.

Ott et al. (2004) show that rotation increases the amplitude of GW because of the induced anisotropy. But if rotation is really fast, for $\beta=E_{\mathrm{rot}} /\left|E_{\mathrm{pot}}\right|>0.3 \%$, the amplitude of $\mathrm{GW}$ becomes smaller, and the characteristic frequency is shifted to lower frequencies. Except for the $85 M_{\odot}$ star, our models have a high $\beta$ so their GW emission is expected to be damped by their high rotation rate. Suwa et al. (2007) show that very massive collapsing PopIII stars may contribute to the GW background and be detectable by future facilities such as DECIGO, the successor of LIGO. In that framework, our $85 M_{\odot}$ model, with $\beta=0.3 \%$, is expected to contribute to this background.

\section{Chemical evolution of the Galaxy with $Z=0$ yields}

Yields of extremely low-metallicity models are constrained by the most metal-poor stars observed in the halo of our Galaxy. They are supposed to trace the local chemical evolution of their surrounding medium at the time of their birth. The notion of time is important because stars with different masses have different lifetimes; the elements they produce are not released at the same moment. The oldest stars observed nowadays may have formed when only massive stars had time to contribute to the enrichment of the medium. If we want to understand the abundances observed at the surface of these old stars, we cannot just integrate the yields obtained by a set of models with an IMF, because we would lose track of the time. What we need is a complete chemical evolution model (CEM).

Such a CEM is computed by Chiappini et al. (2006b) for example ${ }^{6}$. They compare their predictions for the evolution of $\mathrm{N} / \mathrm{O}$ and $\mathrm{C} / \mathrm{O}$ upon the adoption of different sets of stellar yields

\footnotetext{
6 The details of the models can be found in Chiappini et al. (2006a), where they show that such a model reproduces the metallicity distribution of the Galactic halo stars. This means that the timescale for the chemical enrichment in the halo is well constrained.
}

with the surface ratios determined for the metal-poor stars observed by Israelian et al. (2004) and Spite et al. (2005). They show that the N/O ratio is much higher than what was predicted by a CEM using the yields of the slow-rotating $Z=10^{-5}$ models from Meynet \& Maeder (2002) down to $Z=0$. When adding the yields of the fast-rotating $Z=10^{-8}$ models from Hirschi (2007), the fit is much improved. The same improvement is found for the $\mathrm{C} / \mathrm{O}$ ratio, which presents an upturn at low metallicity. Note that the high $\mathrm{N} / \mathrm{O}$ and the $\mathrm{C} / \mathrm{O}$ upturn of the low-metallicity stars is also observed in low-metallicity DLAs (Pettini et al. 2008).

Their CEM is computed with the assumption that yields from $Z=10^{-8}$ stellar models are valid down to $Z=0$. But actually, there is a physical transition at $Z_{\text {trans }} \simeq 10^{-10}$ : the stars below $Z_{\text {trans }}$ present a PopIII-like behaviour (with some He burning already during the MS), and the stars above $Z_{\text {trans }}$ have well separated $\mathrm{H}$ - and He-burning phases.

We computed a similar CEM, but this time including our $Z=0$ pre-SN yields up to $Z_{\text {trans }}$, the yields of Hirschi (2007) up to $Z=10^{-5}$, and then the yields from Meynet \& Maeder (2002) and Hirschi et al. (2004). For the PopIII stellar models, we used an IMF that is truncated at $1 M_{\odot}$ but is otherwise standard. Compared to the chemical evolution computed using the $Z=10^{-8}$ yields down to $Z=0$ (Fig. 9, left panel), the new computation predicts a reduction of the $\mathrm{N} / \mathrm{O}$ and $\mathrm{C} / \mathrm{O}$ ratios at extremely low metallicity $\left(Z \leq 6 \times 10^{-7}\right)$. This trend is expected given the higher $\mathrm{O}$ and the lower $\mathrm{C}$ and $\mathrm{N}$ production of the PopIII models (Sect. 4.2 and Fig. 7). Note that a similar CEM computed with the "wind-only" contribution for our 25 to $60 M_{\odot}$ models leads to such a slow $\mathrm{O}$ enrichment that it would deviate from the CEM with $Z=10^{-8}$ yield curve only at an $\mathrm{O} / \mathrm{H}$ domain much lower than that shown in Fig. 9.

Another interesting quantity is the ratio ${ }^{12} \mathrm{C} /{ }^{13} \mathrm{C}$. A large $\mathrm{N}$ production comes with a high ${ }^{13} \mathrm{C}$ value since they are both the products of ${ }^{12} \mathrm{C}$ conversion by the $\mathrm{CN}$-cycle in $\mathrm{H}$-burning zones. We thus expect a low ${ }^{12} \mathrm{C} /{ }^{13} \mathrm{C}$ ratio from the yields of 

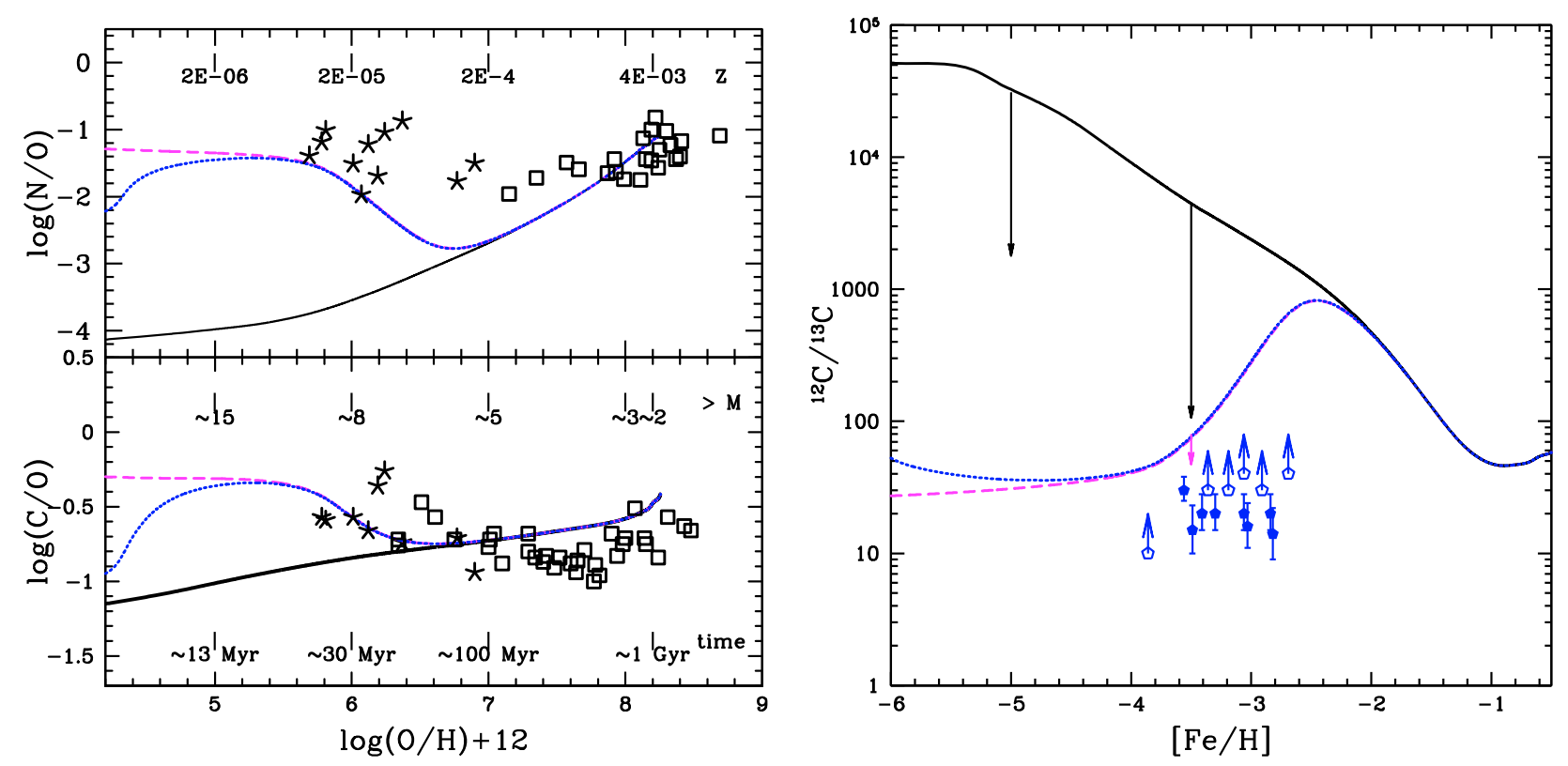

Fig. 9. Solid (black) curve: the CEM obtained with the stellar yields of slow rotating $Z=10^{-5}$ models from Meynet \& Maeder (2002) and Hirschi et al. (2004). Dashed (magenta) line includes the yields of fast rotating $Z=10^{-8}$ models from Hirschi (2007) at very low metallicity. Dotted (blue) curve is obtained using the yields of the $Z=0$ models presented in this paper up to $Z=10^{-10}$. Left: evolution of the N/O and C/O ratios. Data points are from Israelian et al. (2004, open squares) and Spite et al. (2005, stars). Right: evolution of the ${ }^{12} \mathrm{C} /{ }^{13} \mathrm{C}$ ratio. Data points are unmixed stars from Spite et al. (2006): open pentagons are lower limits. The arrows going down from the theoretical curves indicate the final ${ }^{12} \mathrm{C} /{ }^{13} \mathrm{C}$ observed in giants (after the dredge-up), starting from the initial composition values given by the stellar models (see Chiappini et al. 2008).

fast-rotating models. Chiappini et al. (2008) use the same set of yields as in Chiappini et al. (2006b), but this time to study the ${ }^{12} \mathrm{C} /{ }^{13} \mathrm{C}$ ratio. They show that this ratio is indeed much closer to the recent observations provided by Spite et al. (2006) upon the inclusion of fast-rotating $Z=10^{-8}$ models. With the inclusion of our pre-SN PopIII yields (Fig. 9, right panel), we predict that the ${ }^{12} \mathrm{C} /{ }^{13} \mathrm{C}$ ratio should rise slightly again with decreasing $[\mathrm{Fe} / \mathrm{H}]$ at extremely low metallicity.

\section{Discussion and conclusion}

We computed a set of metal-free stellar models with masses between 9 and $200 M_{\odot}$, following their evolution until the presupernova stage. The set was composed of seven differentially rotating models with an initial velocity of $800 \mathrm{~km} \mathrm{~s}^{-1}$ (except for the $9 M_{\odot}$ model which started the main sequence with $500 \mathrm{~km} \mathrm{~s}^{-1}$ ), and a comparison set of non-rotating models. We showed that in PopIII stars, although the effects of rotation are important, they are less strong than in stars with even a tiny amount of metals $\left(Z=10^{-8}\right)$. There are two main causes for this:

1. The meridional circulation is extremely weak at $Z=0$. The dependance of the outer cell in $1 / \rho$ (see Sect. 3.3) makes it a factor of 100 lower compared to the value at standard metallicity, and still a factor of 4 lower compared to $Z=10^{-5}$. The evolution of the angular momentum is close to local conservation, so the critical limit is reached late. Moreover, only a very small amount of mass needs to be lost in order to bring the surface back to sub-critical rotation.

2. There is little structural readjustment at the end of the MS, because the core is already hot enough to burn some He during the main sequence, so the nuclear burning is continuous. The transition between $\mathrm{H}$ and He burning is smooth and the models remain on the blue side of the Hertzsprung-Russell diagram for a long part of the core He burning. When they eventually reach the red side, their outer convective zone remains thin, so the dredge-up is not efficient and the surface enrichment stays low. The envelope remains transparent to radiation and the mass loss is negligible.

There is actually a mass loss where none was expected (because of the wind dependance on $Z$ ), but it remains modest even for the most massive of our models.

Could this picture be modified by changing the physical inputs in the models? We have shown that the situation is qualitatively the same when we take a faster initial rotation rate. The main problem is the weakness of the meridional circulation, which does not efficiently feed the outer layers with angular momentum taken from the fast spinning core. So, even if the critical limit is reached a little earlier, the models still do not lose a lot of mass. For this work, we did not take into account the anisotropy of the winds in our models. We do not think it would change the picture presented here, because the loss of angular momentum by radiative mass loss is negligible. There is still an interesting possibility: the implementation of magnetic fields. They have been shown to change many features in stellar evolution (Heger et al. 2005; Maeder \& Meynet 2005), and to provide the strong core-envelope coupling that is lacking in the $Z=0$ models. With such a coupling, the mass loss becomes much larger and the evolution may be strongly altered (Ekström et al. 2008a).

With the initial velocities considered here, our models do not follow a chemically homogeneous evolution (Maeder 1987; Heger \& Langer 2000). In the frame of the physics we used, homogeneous evolution for Pop III stars would need faster rotation rates. When the magnetic dynamo mechanism proposed by Spruit $(1999,2002)$ is taken into account, chemical mixing becomes more efficient and homogeneous evolution can be achieved with smaller initial rotation rates (Yoon \& Langer 2005; Yoon et al. 2006; Woosley \& Heger 2006). Note however that at extremely low metallicities, the weakening of the 
meridional circulation becomes a real handicap for the models to evolve chemically homogeneously. Hirschi et al. (2008) find that at $Z=10^{-8}$, a $40 M_{\odot}$ model (computed with magnetic fields) starting its evolution with $v / v_{\text {crit }}=0.55$ does not rotate fast enough to follow such an homogeneous evolution. We expect that at $Z=0$ the problem would be still more acute. The consequences of such extreme effects of rotation is left for a future work.

The only prescription for a stellar dynamo existing to date, the Tayler-Spruit formalism (Spruit 1999, 2002), is still debated: recent works have cast some doubts on the efficiency of the Tayler-Spruit dynamo mechanism. For instance, Zahn et al. (2007) have shown that although the Pitts and Tayler instability indeed develops, it does not succeed in driving a dynamo. Moreover, this dynamo needs a pre-existing magnetic-field seed, and it is not yet clear whether magnetic fields were present in the early Universe or not (see Giovannini 2004, for a detailed discussion on this subject).

We presented pre-supernova yields for our stellar models. We included them in a chemical evolution model of the Galactic halo. Our results predict that the N/O and the $\mathrm{C} / \mathrm{O}$ ratios should drop at extremely low metallicity $\left(Z \leq 10^{-6}\right)$. For the time being though, the observational discrimination between the two curves is far beyond our observational possibilities. We also gave the rotational characteristics of the iron core of our models and showed that since they do not lose much angular momentum during their evolution, they keep high rotation rates in the core, so their final explosion may be much more energetic than sometimes assumed. We showed that the most massive of our models may contribute to the emission of the gravitational wave background.

Many interesting questions and issues were not treated in this study. Our mass coverage was too coarse to accurately constrain the limiting masses for undergoing a CNO shell boost, for example, or for the different fates at the end of the evolution. Even without knowing if magnetic fields were indeed present in the early Universe, it would be interesting to study the role they could play in primordial stellar evolution. As mentioned above, they could drastically change the picture presented here. We have also left for a forthcoming paper the question of reionisation. Rotation changes the luminosity, the $T_{\text {eff }}$ and the lifetimes of the models, so it should change the ionising power of the first stars.

\section{References}

Abel, T., Bryan, G. L., \& Norman, M. L. 2002, Science, 295, 93 Alexander, D. R., \& Ferguson, J. W. 1994, ApJ, 437, 879

Aoki, W., Beers, T. C., Christlieb, N., et al. 2007, ApJ, 655, 492

Barklem, P. S., Christlieb, N., Beers, T. C., et al. 2005, A\&A, 439, 129

Bromm, V., Coppi, P. S., \& Larson, R. B. 2002, ApJ, 564, 23

Cayrel, R., Depagne, E., Spite, M., et al. 2004, A\&A, 416, 1117

Chaboyer, B., \& Zahn, J.-P. 1992, A\&A, 253, 173

Chiappini, C., Hirschi, R., Matteucci, F., et al. 2006a, in International Symposium on Nuclear Astrophysics - Nuclei in the Cosmos Chiappini, C., Hirschi, R., Meynet, G., et al. 2006b, A\&A, 449, L27 Chiappini, C., Ekström, S., Meynet, G., et al. 2008, A\&A, 479, L9 Chieffi, A., \& Limongi, M. 2004, ApJ, 608, 405

Chieffi, A., Domínguez, I., Limongi, M., \& Straniero, O. 2001, ApJ, 554, 1159 Christlieb, N., Bessell, M. S., Beers, T. C., et al. 2002, Nature, 419, 904 Cohen, J. G., Christlieb, N., McWilliam, A., et al. 2007, ArXiv e-prints, 709 Ekström, S., Meynet, G., \& Maeder, A. 2008a, in Massive Stars as Cosmic Engines, in press, ed. F. Bresolin, P. Crowther, \& J. Puls, IAU Symp., 250, Ekström, S., Meynet, G., Maeder, A., \& Barblan, F. 2008b, A\&A, 478, 467 Eldridge, J. J., \& Tout, C. A. 2004, MNRAS, 353, 87

Fryer, C. L., Woosley, S. E., \& Heger, A. 2001, ApJ, 550, 372

Gil-Pons, P., Suda, T., Fujimoto, M. Y., \& García-Berro, E. 2005, A\&A, 433, 1037
Gil-Pons, P., Gutiérrez, J., \& García-Berro, E. 2007, A\&A, 464, 667

Giovannini, M. 2004, Int. J. Mod. Phys. D, 13, 391

Greif, T. H., \& Bromm, V. 2006, MNRAS, 373, 128

Heger, A., Fryer, C. L., Woosley, S. E., Langer, N., \& Hartmann, D. H. 2003, ApJ, 591, 288

Heger, A., \& Langer, N. 2000, ApJ, 544, 1016

Heger, A., \& Woosley, S. E. 2002, ApJ, 567, 532

Heger, A., Langer, N., \& Woosley, S. E. 2000a, ApJ, 528, 368

Heger, A., Woosley, S. E., \& Waters, R. 2000b, in The First Stars: Proceedings of the MPA/ESO Workshop Held at Garching, Germany, 4-6 August 1999, ed. A. Weiss, T. G. Abel, \& V. Hill (Springer Verlag), 121

Heger, A., Woosley, S. E., \& Spruit, H. C. 2005, ApJ, 626, 350

Hirschi, R. 2007, A\&A, 461, 571

Hirschi, R., Meynet, G., \& Maeder, A. 2004, A\&A, 425, 649

Hirschi, R., Chiappini, C., Meynet, G., Maeder, A., \& Ekström, S. 2008, in IAU Symp., 250, 217

Iglesias, C. A., \& Rogers, F. J. 1996, ApJ, 464, 943

Iocco, F., Mangano, G., Miele, G., Pisanti, O., \& Serpico, P. D. 2007, Phys. Rev. D, 75, 087304

Israelian, G., Ecuvillon, A., Rebolo, R., et al. 2004, A\&A, 421, 649

Kudritzki, R. P. 2002, ApJ, 577, 389

Lau, H. H. B., Stancliffe, R. J., \& Tout, C. A. 2008, MNRAS, 174

Limongi, M., \& Chieffi, A. 2002, Publ. Astron. Soc. Austr., 19, 246

Limongi, M., Chieffi, A., \& Straniero, O. 2001, Mem. Soc. Astron. Ital., 72, 289

Limongi, M., Straniero, O., \& Chieffi, A. 2000, ApJS, 129, 625

Maeder, A. 1987, A\&A, 178, 159

Maeder, A. 1992, A\&A, 264, 105

Maeder, A. 1997, A\&A, 321, 134

Maeder, A., \& Meynet, G. 2000a, A\&A, 361, 159

Maeder, A., \& Meynet, G. 2000b, ARA\&A, 38, 143

Maeder, A., \& Meynet, G. 2001, A\&A, 373, 555

Maeder, A., \& Meynet, G. 2005, A\&A, 440, 1041

Maeder, A., \& Zahn, J.-P. 1998, A\&A, 334, 1000

Marigo, P., Girardi, L., Chiosi, C., \& Wood, P. R. 2001, A\&A, 371, 152

Marigo, P., Chiosi, C., \& Kudritzki, R.-P. 2003, A\&A, 399, 617

Meynet, G., Ekström, S., \& Maeder, A. 2006, A\&A, 447, 623

Meynet, G., \& Maeder, A. 2000, A\&A, 361, 101

Meynet, G., \& Maeder, A. 2002, A\&A, 390, 561

Meynet, G., \& Maeder, A. 2003, A\&A, 404, 975

Meynet, G., \& Maeder, A. 2005, A\&A, 429, 581

Nakamura, F., \& Umemura, M. 2001, ApJ, 548, 19

Nomoto, K. 1984, in Stellar Nucleosynthesis, Proceedings of the 3rd. Workshop of the Advanced School of Astronomy of the Ettore Majorana Centre for Scientific Culture, Erice, Italy, May 11-21, 1983, ed. C. Chiosi, \& A. Renzini, 239

Omukai, K., \& Yoshii, Y. 2003, ApJ, 599, 746

O'Shea, B. W., Abel, T., Whalen, D., \& Norman, M. L. 2005, ApJ, 628, L5

Ott, C. D., Burrows, A., Livne, E., \& Walder, R. 2004, ApJ, 600, 834

Owocki, S. 2005, in The Nature and Evolution of Disks Around Hot Stars, ed. R. Ignace, \& K. G. Gayley, ASP Conf. Ser., 337, 101

Pettini, M., Zych, B. J., Steidel, C. C., \& Chaffee, F. H. 2008, MNRAS, in press Salvadori, S., Schneider, R., \& Ferrara, A. 2007, MNRAS, 381, 647

Scannapieco, E., Madau, P., Woosley, S., Heger, A., \& Ferrara, A. 2005, ApJ, 633,1031

Shapiro, S. L., \& Teukolsky, S. A. 1983, Black holes, white dwarfs, and neutron stars: the physics of compact objects, Research supported by the National Science Foundation (New York: Wiley-Interscience), 663

Shigeyama, T., Tsujimoto, T., \& Yoshii, Y. 2003, ApJ, 586, L57

Shimizu, T. M., Ebisuzaki, T., Sato, K., \& Yamada, S. 2001, ApJ, 552, 756

Siess, L., Livio, M., \& Lattanzio, J. 2002, ApJ, 570, 329

Silk, J., \& Langer, M. 2006, MNRAS, 371, 444

Spite, M., Cayrel, R., Plez, B., et al. 2005, A\&A, 430, 655

Spite, M., Cayrel, R., Hill, V., et al. 2006, A\&A, 455, 291

Spruit, H. C. 1999, A\&A, 349, 189

Spruit, H. C. 2002, A\&A, 381, 923

Suwa, Y., Takiwaki, T., Kotake, K., \& Sato, K. 2007, ApJ, 665, L43

Talon, S., \& Zahn, J.-P. 1997, A\&A, 317, 749

Umeda, H., \& Nomoto, K. 2003, Nature, 422, 871

Umeda, H., \& Nomoto, K. 2005, ApJ, 619, 427

Walder, R., Burrows, A., Ott, C. D., et al. 2005, ApJ, 626, 317

Woosley, S. E. 1993, ApJ, 405, 273

Woosley, S. E., \& Heger, A. 2006, ApJ, 637, 914

Yamasaki, T., \& Yamada, S. 2005, ApJ, 623, 1000

Yoon, S.-C., \& Langer, N. 2005, A\&A, 443, 643

Yoon, S.-C., Langer, N., \& Norman, C. 2006, A\&A, 460, 199

Zahn, J.-P. 1992, A\&A, 265, 115

Zahn, J.-P., Brun, A. S., \& Mathis, S. 2007, A\&A, 474, 145 